\title{
On non fundamental group equivalent surfaces
}

\author{
MINA TEICHER \\ MICHAEL FRIEDMAN
}

\begin{abstract}
In this paper we present an example of two polarized $\mathrm{K} 3$ surfaces which are not Fundamental Group Equivalent (their fundamental groups of the complement of the branch curves are not isomorphic; denoted by FGE) but the fundamental groups of their related Galois covers are isomorphic. For each surface, we consider a generic projection to $\mathbb{C P}^{2}$ and a degenerations of the surface into a union of planes - the "pillow" degeneration for the non-prime surface and the "magician" degeneration for the prime surface. We compute the Braid Monodromy Factorization (BMF) of the branch curve of each projected surface, using the related degenerations. By these factorizations, we compute the above fundamental groups. It is known that the two surfaces are not in the same component of the Hilbert scheme of linearly embedded K3 surfaces. Here we prove that furthermore they are not FGE equivalent, and thus they are not of the same Braid Monodromy Type (BMT) (which implies that they are not a projective deformation of each other).
\end{abstract}

14J28, 14H30; 14H20, 57M12, 20F36, 14F35, 14Q05

\section{Introduction}

Given $X \subset \mathbb{C P}^{n}$ a smooth algebraic surface of degree $m$, one can obtain information on $X$ by considering it as a branched cover of $\mathbb{C} \mathbb{P}^{2}$. It is well-known that for $X \longrightarrow \mathbb{C P}^{2}$ a generic projection, the branch locus is a plane curve $\bar{S} \subset \mathbb{C P}^{2}$ which is, in general, singular, and its singularities are nodes and cusps. Let $S \subset \mathbb{C}^{2} \subset \mathbb{C P}^{2}$ be a generic affine portion of $\bar{S}$.

It was proven in Kulikov-Teicher [8] that if the Braid Monodromy Factorizations (BMF) of the branch loci of two surfaces $X_{1}$ and $X_{2}$ are Hurwitz-equivalent, then the surfaces are diffeomorphic. Moreover, if the factorizations are not Hurwitz-equivalent, then $X_{1}$ and $X_{2}$ are not projectively deformation equivalent. Therefore, the BMT invariant (the equivalence class of a BMF) is really in the "middle", ie, between the diffeomorphism equivalence and the projectively deformation equivalence. We need to find an algorithm that decides whether two BMFs are equivalent. In general, it was shown in LibermanTeicher [9] that there is no finite algorithm which determines whether two positive 
factorizations are Hurwitz- equivalent. However, [9] did not examine the particular case of the BMFs. Therefore, we have to extract the information contained in the braid monodromy factorization via the introduction of more manageable (but less powerful) invariants.

Two discrete invariants are induced from the BMF of the branch curve $-S$ : the fundamental group of the complement of the branch curve (see Amram-FriedmanTeicher [2], Friedman-Teicher [7], Moishezon [11], Moishezon-Teicher [17]) and its subquotient: the fundamental group of the Galois Cover of $X$ (see Liedtke [10], Moishezon-Robb-Teicher [12], Moishezon-Teicher [13]). We say that two surfaces are Fundamental Group Equivalent (FGE) if their fundamental groups of the complement of the branch curve are isomorphic.

In this article we present two surfaces, which are embeddings of a $\mathrm{K} 3$ surface with respect to two different linear systems; therefore they are diffeomorphic. Due to the nature of the particular linear systems, these embedded surfaces are not projectively deformation equivalent. It is also known that any two K3 surfaces can be abstractly deformed one into the other. Thus one can raise the questions: Are the surfaces FGE? Are the fundamental groups of the corresponding Galois covers isomorphic? Here we prove that although the latter groups are isomorphic, the surfaces are not FGE. Therefore, these surfaces are also not BMT-equivalent, which means that the surfaces are not in the same component of the Hilbert scheme of linearly embedded K3 surfaces.

\section{Acknowledgments}

We are very grateful to Ciro Ciliberto, for pointing out the degeneration used for the "magician" K3 surface and for other helpful discussions. We also wish to thank Meirav Amram for her fruitful remarks.

This work is partially supported by the Emmy Noether Research Institute for Mathematics and the Minerva Foundation of Germany and the Israel Science Foundation grant \# 8008/02-3 (Excellency Center "Group Theoretic Methods in the Study of Algebraic Varieties").

\section{Preliminaries: The $\mathrm{K} 3$ surfaces and the BMT invariant}

In this section we recall the main definitions and constructions regarding the two embeddings of the $K 3$ surface, and the braid monodromy factorization (BMF) related to a (branch) curve. We begin with the introduction of the two embeddings of a $K 3$ surface. 


\subsection{Two embeddings of a $K 3$ surface}

Recall that the surfaces with Kodaira dimension which equals to 0, that are simply connected, have in fact trivial canonical bundle, and are called $K 3$ surfaces. The invariants for such surfaces are $p_{g}=1, q=0, e=24$. The moduli space of all $K 3$ surfaces is 20 -dimensional.

Most $K 3$ surfaces are not algebraic; the algebraic ones are classified by an infinite collection (depending on an integer $g \geq 2$ ) of 19-dimensional moduli spaces. The general member of the family has a rank one Picard group, generated by an ample class $H$ with $H^{2}=2 g-2$; the general member of the linear system $|H|$ is a smooth curve of genus $g$, and this linear system maps the $K 3$ surface to $\mathbb{P}^{g}$ as a surface of degree $2 g-2$. For example, a $K 3$ surface is a smooth quartic surface in $\mathbb{P}^{3}$. The quartic surfaces in $\mathbb{P}^{3}$ form the family with $g=3$. The integer $g$ is called the genus of the family.

The first embedded surface is a $K 3$ surface of genus 9 , embedded in $\mathbb{C P} P^{9}$ by the pillow (2,2)-pillow degeneration (see Ciliberto-Miranda-Teicher [6] for details). The resulting embedding can be degenerated into a union of 16 planes, such that the whole degenerated object would "resemble a pillow" (see Figure 1 for clarification). We denote by $X_{1}$ the embedded $K 3$ surface, and by $\left(X_{1}\right)_{0}$ the degenerated surface (see [17] for an explicit definition of a degeneration).

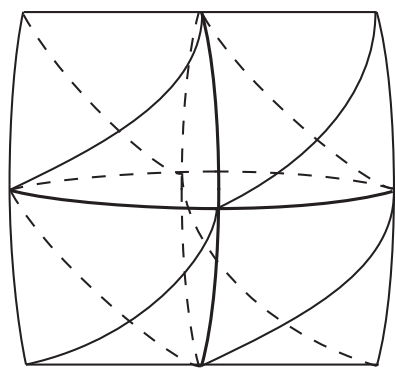

Figure 1: $\left(X_{1}\right)_{0}$ - the $(2,2)-$ pillow degeneration: every triangle denotes a plane

The degeneration process has a "local inverse" - the regeneration process (see an explanation in the following subsection), and for it we need to fix a numeration of vertices (and the lines; see Amram-Ciliberto-Miranda-Teicher [1] for details). This is done as shown in Figure 3.

The 16 planes meet each other along a total of 24 lines, each joining 2 of the 10 coordinate points. We numerate the lines as follows: if $L$ has endpoints $a<b$ and $M$ has endpoints $c<d$, then $L<M$ if $b<d$ or $b=d$ and $a<c$. This gives a total 

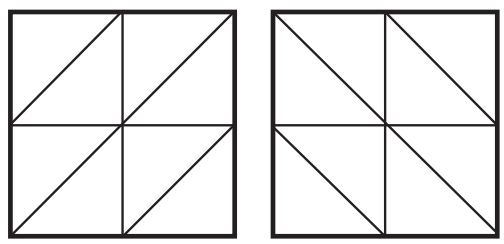

Figure 2: A 2-dimensional figure of $\left(X_{1}\right)_{0}$ : the boundaries are identified (top to top, bottom to bottom, side to side)

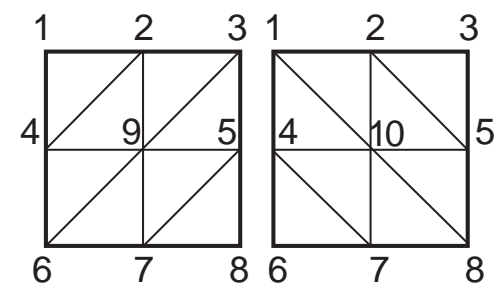

Figure 3: The numeration of the singular points of $\left(X_{1}\right)_{0}$

ordering of the lines, which we interpret as a numbering from 1 to 24 , as shown in Figure 4.

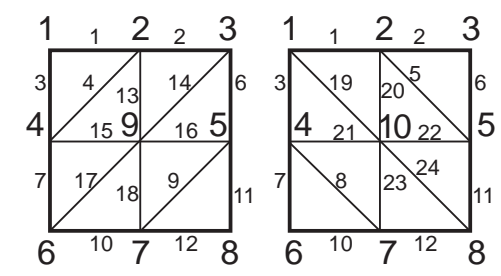

Figure 4: The numeration of the intersection lines of $\left(X_{1}\right)_{0}$

Under a general projection $\pi_{1}:\left(X_{1}\right)_{0} \rightarrow \mathbb{C P}^{2}$, each of the 16 planes is mapped isomorphically to $\mathbb{C P}^{2}$. The ramification locus $R_{1}$ of $\pi_{1}$ is a local isomorphism. Here $R_{1}$ is exactly the 24 lines. Let $\left(S_{1}\right)_{0}=\pi_{1}\left(R_{1}\right)$ be the degenerated branch curve. It is a line arrangement, composed of the image of the 24 lines.

The second embedded surface is also an embedded $K 3$ surface of genus 9 in $\mathbb{C P}^{9}$. We call this surface the "magician" surface, since its degeneration "resembles" a magician's hat. The surface and its degeneration into a union of 16 planes are described in CilibertoMiranda [5]. The dual graph of the degenerated surface is presented explicitly in [5, pg. 430] - and from it we can build the degenerated surface (see Figure 5). 


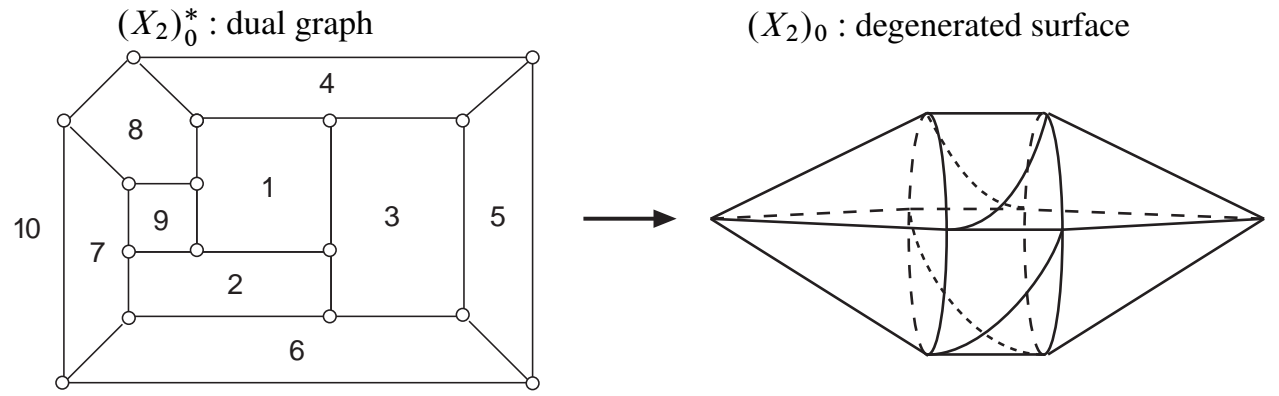

Figure 5: Every point in the dual graph represents a plane; every plane represents a point

Denote by $X_{2}$ this embedded surface, and by $\left(X_{2}\right)_{0}$ the degenerated surface. We can depict a 2-dimensional graph of $\left(X_{2}\right)_{0}$, where the boundaries are identified (see Figure $6)$.

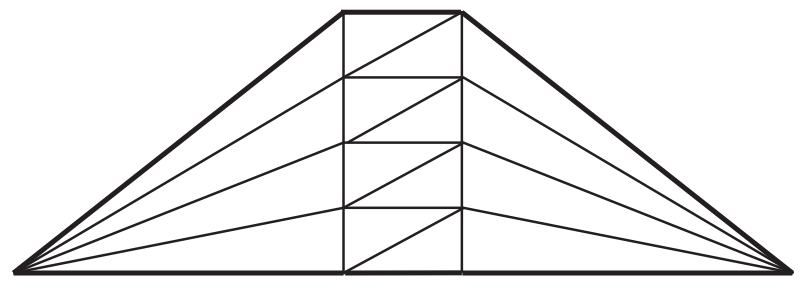

Figure 6: $\left(X_{2}\right)_{0}$ - the boundaries are identified (top to bottom)

Once again, we numerate the vertices and then the edges. We note that the extreme edges of the graph $\left(X_{2}\right)_{0}$ are actually 4-points: singular points in the degenerated surface which are the intersection of four planes. In order to regenerate it (see Robb [19] for the possible degenerations of this point), we need to numerate the vertices in such a way that the number of "entering" and "exiting" lines from these points will be equal. Therefore, we numerate them as vertices 5 and 6 . Following the symmetry appearing in the graph, we numerate the other vertices as follows (see Figure 7).

Note that $\left(X_{2}\right)_{0}$ also contains 24 intersection lines and 10 singular points. We denote by $\left(S_{2}\right)_{0}=\pi_{2}\left(R_{2}\right)$ the degenerated branch curve with respect to a generic projection $\pi_{2}:\left(X_{2}\right)_{0} \rightarrow \mathbb{C P}^{2}$.

Since every two $K 3$ surfaces are diffeomorphic, $X_{1}$ and $X_{2}$ are also diffeomorphic. Note that the Hilbert scheme of embedded linearly normal K3 surfaces can be reducible. This is indeed the case here - the Picard group Pic $X_{1}$ is generated by $\frac{1}{2} H$ (where $H$ 


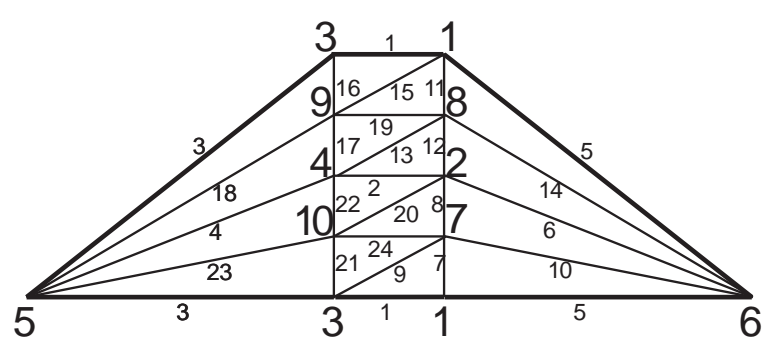

Figure 7: Numeration of $\left(X_{2}\right)_{0}$

is the hyperplane class; see Ciliberto-Miranda-Teicher [6]) and Pic $X_{2}$ is generated by $H$ (see Ciliberto-Miranda [5]).

Two polarized K3 surfaces are projectively deformation equivalent if and only if there is a diffeomorphism which carries the hyperplane class to the hyperplane class. As indicated above, this is not the case. We show in the following sections that these surfaces are also not BMT-equivalent, and that the fundamental groups of complement of the branch curve can also be used in order to differentiate between irreducible components of the Hilbert scheme. Thus it is a topological invariant that arises in algebro-geometric considerations.

\subsection{The braid group and the BMF}

Recall that computing the braid monodromy is the main tool to compute fundamental groups of complements of curves. The reader who is familiar with this subject can skip the following definitions. We begin by defining the braid monodromy associated to a curve.

Let $D$ be a closed disk in $\mathbb{R}^{2}, K \subset \operatorname{Int}(D), K$ finite, $n=\# K$. Recall that the braid group $B_{n}[D, K]$ can be defined as the group of all equivalent diffeomorphisms $\beta$ of $D$ such that $\beta(K)=K,\left.\beta\right|_{\partial D}=\left.\operatorname{Id}\right|_{\partial D}$.

Definition ( $H(\sigma)$, half-twist defined by $\sigma)$ Let $a, b \in K$, and let $\sigma$ be a smooth simple path in $\operatorname{Int}(D)$ connecting $a$ with $b$ s.t. $\sigma \cap K=\{a, b\}$. Choose a small regular neighborhood $U$ of $\sigma$ contained in $\operatorname{Int}(D)$, s.t. $U \cap K=\{a, b\}$. Denote by $H(\sigma)$ the diffeomorphism of $D$ which switches $a$ and $b$ by a counterclockwise $180^{\circ}$ rotation and is the identity on $D \backslash U$. Thus it defines an element of $B_{n}[D, K]$, called the half-twist defined by $\sigma$.

Denote $[A, B]=A B A^{-1} B^{-1},\langle A, B\rangle=A B A B^{-1} A^{-1} B^{-1}$. We recall the Artin presentation of the braid group. 
Theorem 2.1 $B_{n}$ is generated by the half-twists $H_{i}$ of a frame $H_{i}$ and all the relations between $H_{1}, \ldots, H_{n-1}$ follow from

$$
\begin{gathered}
{\left[H_{i}, H_{j}\right]=1 \text { if }|i-j|>1} \\
\left\langle H_{i}, H_{j}\right\rangle=1 \text { if }|i-j|=1 .
\end{gathered}
$$

Assume that all of the points of $K$ are on the $X$-axis (when considering $D$ in $\mathbb{R}^{2}$ ). In this situation, if $a, b \in K$, and $z_{a, b}$ is a path that connects them, then we denote it by $Z_{a, b}=H\left(z_{a, b}\right)$. If $z_{a, b}$ is a path that goes below the $X$-axis, then we denote it by $\underline{Z}_{a, b}$, or just $Z_{a, b}$. If $z_{a, b}$ is a path that goes above the $x$-axis, then we denote it by $\bar{Z}_{a, b}$. We also denote by $\underline{Z}_{a, b}^{(c-d)}\left(\underset{(c-d)}{\left(\bar{Z}_{a, b}\right)}\right)$ the braid induced from a path connecting the points $a$ and $b$ below (resp. above) the $X$-axis, going above (resp. below) it from the point $c$ till point $d$.

Definition (The braid monodromy w.r.t. $S, \pi, u$ ) Let $S$ be a curve, $S \subseteq \mathbb{C}^{2}$. Let $\pi: S \rightarrow \mathbb{C}^{1}$ be defined by $\pi(x, y)=x$. We denote deg $\pi$ by $m$. Let $N=\left\{x \in \mathbb{C}^{1} \mid\right.$ $\left.\# \pi^{-1}(x)<m\right\}$. Take $u \notin N$, s.t. $\Re(x) \ll u \quad \forall x \in N$. Let $\mathbb{C}_{u}^{1}=\{(u, y)\}$. There is a naturally defined homomorphism

$$
\pi_{1}\left(\mathbb{C}^{1}-N, u\right) \stackrel{\varphi}{\rightarrow} B_{m}\left[\mathbb{C}_{u}^{1}, \mathbb{C}_{u}^{1} \cap S\right]
$$

which is called the braid monodromy w.r.t. $S, \pi, u$, where $B_{m}$ is the braid group. We sometimes denote $\varphi$ by $\varphi_{u}$. In fact, denoting by $E$, a big disk in $\mathbb{C}^{1}$ s.t. $E \supset N$, we can also take the path in $E \backslash N$ not to be a loop, but just a non-self-intersecting path. This induces a diffeomorphism between the models $(D, K)$ at the two ends of the considered path, where $D$ is a big disk in $\mathbb{C}_{u}^{1}$, and $K=\mathbb{C}_{u}^{1} \cap S \subset D$.

Definition $\left(\psi_{T}\right.$, Lefschetz diffeomorphism induced by a path $\left.T\right)$ Let $T$ be a path in $E \backslash N$ connecting $x_{0}$ with $x_{1}, T:[0,1] \rightarrow E \backslash N$. There exists a continuous family of diffeomorphisms $\psi_{(t)}: D \rightarrow D, t \in[0,1]$, such that $\psi_{(0)}=\mathrm{Id}, \psi_{(t)}\left(K\left(x_{0}\right)\right)=$ $K(T(t))$ for all $t \in[0,1]$, and $\psi_{(t)}(y)=y$ for all $y \in \partial D$. For emphasis we write $\psi_{(t)}:\left(D, K\left(x_{0}\right)\right) \rightarrow(D, K(T(t))$. A Lefschetz diffeomorphism induced by a path $T$ is the diffeomorphism

$$
\psi_{T}=\psi_{(1)}:\left(D, K\left(x_{0}\right)\right) \longrightarrow\left(D, K\left(x_{1}\right)\right) .
$$

Since $\psi_{(t)}\left(K\left(x_{0}\right)\right)=K(T(t))$ for all $t \in[0,1]$, we have a family of canonical isomorphisms

$$
\psi_{(t)}^{v}: B_{p}\left[D, K\left(x_{0}\right)\right] \longrightarrow B_{p}[D, K(T(t))], \text { for all } t \in[0,1] .
$$


We recall Artin's theorem on the presentation of the Dehn twist of the braid group as a product of braid monodromy elements of a geometric-base (a base of $\pi=\pi\left(\mathbb{C}^{1}-N, u\right)$ with certain properties; see Moishezon-Teicher [14] for definitions).

Theorem 2.2 Let $S$ be a curve transversal to the line in infinity, and $\varphi$ is a braid monodromy of $S, \varphi: \pi \rightarrow B_{m}$. Let $\delta_{i}$ be a geometric (free) base ( $g$-base) of $\pi$, and $\Delta^{2}$ is the generator of Center $\left(B_{m}\right)$. Then:

$$
\Delta^{2}=\prod \varphi\left(\delta_{i}\right)
$$

This product is also defined as the braid monodromy factorization (BMF) related to a curve $S$.

Note that if $x_{1}, \ldots, x_{n-1}$ are the generators of $B_{n}$, then we know that $\Delta^{2}=\left(x_{1} \ldots \ldots\right.$ $\left.x_{n-1}\right)^{n}$ and thus $\operatorname{deg}\left(\Delta^{2}\right)=n(n-1)$.

So in order to find out what is the braid monodromy factorization of $\Delta_{p}^{2}$, we have to find out what are $\varphi\left(\delta_{i}\right), \forall i$. We refer the reader to the definition of a skeleton (see Moishezon-Teicher [15]) $\lambda_{x_{j}}, x_{j} \in N$, which is a model of a set of paths connecting points in the fiber, s.t. all those points coincide when approaching $A_{j}=\left(x_{j}, y_{j}\right) \in S$, when we approach this point from the right. To describe this situation in greater detail, for $x_{j} \in N$, let $x_{j}^{\prime}=x_{j}+\alpha$. So the skeleton in $x_{j}$ is defined as a system of paths connecting the points in $K\left(x_{j}^{\prime}\right) \cap D\left(A_{j}, \varepsilon\right)$ when $0<\alpha \ll \varepsilon \ll 1, D\left(A_{j}, \varepsilon\right)$ is a disk centered in $A_{j}$ with radius $\varepsilon$.

For a given skeleton, we denote by $\Delta\left\langle\lambda_{x_{j}}\right\rangle$ the braid which rotates by $180^{\circ}$ counterclockwise a small neighborhood of the given skeleton. Note that if $\lambda_{x_{j}}$ is a single path, then $\Delta\left\langle\lambda_{x_{j}}\right\rangle=H\left(\lambda_{x_{j}}\right)$.

We also refer the reader to the definition of $\delta_{x_{0}}$, for $x_{0} \in N$ (see [15]), which describes the Lefschetz diffeomorphism induced by a path going below $x_{0}$, for different types of singular points (tangent, node, branch; for example, when going below a node, a half-twist of the skeleton occurs and when going below a tangent point, a full-twist occurs).

We define, for $x_{0} \in N$, the following number: $\varepsilon_{x_{0}}=1,2,4$ when $\left(x_{0}, y_{0}\right)$ is a branch/node/tangent point (respectively). So we have the following statement (see [15, Proposition 1.5]).

Let $\gamma_{j}$ be a path below the real line from $x_{j}$ to $u$, s.t. $\ell\left(\gamma_{j}\right)=\delta_{j}$. So

$$
\varphi_{u}\left(\delta_{j}\right)=\varphi\left(\delta_{j}\right)=\Delta\left\langle\left(\lambda_{x_{j}}\right)\left(\prod_{m=j-1}^{1} \delta_{x_{m}}\right)\right\rangle^{\varepsilon_{x_{j}}} .
$$


When denoting $\xi_{x_{j}}=\left(\lambda_{x_{j}}\right)\left(\prod_{m=j-1}^{1} \delta_{x_{m}}\right)$ we get -

$$
\varphi\left(\delta_{j}\right)=\Delta\left\langle\left(\xi_{x_{j}}\right)\right\rangle^{\varepsilon_{x_{j}}} .
$$

Note that the last formula gives an algorithm to compute the needed factorization.

For a detailed explanation of the braid monodromy, see [14].

We shall now define an equivalence relation on the BMF.

Definition (Hurwitz moves) Let $\vec{t}=\left(t_{1}, \ldots, t_{m}\right) \in G^{m}$. We say that

$$
\vec{s}=\left(s_{1}, \ldots, s_{m}\right) \in G^{m}
$$

is obtained from $\vec{t}$ by the Hurwitz move $R_{k}$ (or $\vec{t}$ is obtained from $\vec{s}$ by the Hurwitz move $R_{k}^{-1}$ ) if

$$
\begin{aligned}
s_{i} & =t_{i} \text { for } i \neq k, k+1, \\
s_{k} & =t_{k} t_{k+1} t_{k}^{-1}, \\
s_{k+1} & =t_{k} .
\end{aligned}
$$

Definition (Hurwitz move on a factorization) Let $G$ be a group $t \in G$. Let $t=$ $t_{1} \cdots \cdot t_{m}=s_{1} \cdots s_{m}$ be two factorized expressions of $t$. We say that $s_{1} \cdots \cdot s_{m}$ is obtained from $t_{1} \cdots t_{m}$ by a Hurwitz move $R_{k}$ if $\left(s_{1}, \ldots, s_{m}\right)$ is obtained from $\left(t_{1}, \ldots, t_{m}\right)$ by a Hurwitz move $R_{k}$.

Definition (Hurwitz equivalence of factorization) Two factorizations are Hurwitz equivalent if they are obtained from each other by a finite sequence of Hurwitz moves.

Definition (Braid monodromy type of curves (BMT)) Two curves $S_{1}$ and $S_{2}$ are of the same BMT (denoted by $\cong$ ) if they have related BMF that are equivalent.

In 1998, the following theorem was proved by Kulikov-Teicher [8].

Theorem 2.3 If $S_{1} \cong S_{2}$, then $S_{1}$ is isotopic to $S_{2}$ (when $S_{1}, S_{2}$ are any curves).

Thus, an invariant of surfaces can be derived from the BMT of the branch curve of a surface.

Definition (Braid monodromy type of surfaces (BMT)) The BMT of a projective surface is the BMT of the branch curve of a generic projection of the surface embedded in a projective space by means of a complete linear system. 
Consequently, the following was proved [8].

Theorem 2.4 The BMT of a projective surface $X$ determines the diffeomorphism type of $X$.

We recall now the regeneration methods.

The regeneration methods are actually, locally, the reverse process of the degeneration method. When regenerating a singular configuration consisting of lines and conics, the final stage in the regeneration process involves doubling each line, so that each point of $K$ corresponding to a line labelled $i$ is replaced by a pair of points, labelled $i$ and $i^{\prime}$. The purpose of the regeneration rules is to explain how the braid monodromy behaves when lines are doubled in this manner. We denote by $Z_{i, j}=H\left(z_{i, j}\right)$ where $z_{i, j}$ is a path connecting points in $K$.

The rules are (see Moishezon-Teicher [16, pages 336-337]):

(i) First regeneration rule (the regeneration of a branch point of any conic): A factor of the braid monodromy of the form $Z_{i, j}$ is replaced in the regeneration by $Z_{i^{\prime}, j} \cdot \underline{(j)}_{i, j^{\prime}}$.

(ii) Second regeneration rule (the regeneration of a node): A factor of the form $Z_{i j}^{2}$ is replaced by a factorized expression $Z_{i i^{\prime}, j}^{2}:=Z_{i^{\prime} j}^{2} \cdot Z_{i j}^{2}, Z_{i, j j^{\prime}}^{2}:=Z_{i j^{\prime}}^{2} \cdot Z_{i j}^{2}$ or by $Z_{i i^{\prime}, j j^{\prime}}^{2}:=Z_{i^{\prime} j^{\prime}}^{2} \cdot Z_{i j^{\prime}}^{2} Z_{i^{\prime} j}^{2} \cdot Z_{i j}^{2}$.

(iii) Third regeneration rule (the regeneration of a tangent point): A factor of the form $Z_{i j}^{4}$ in the braid monodromy factorized expression is replaced by $Z_{i, j j^{\prime}}^{3}:=\left(Z_{i j}^{3}\right)^{Z_{j j^{\prime}}} \cdot\left(Z_{i j}^{3}\right) \cdot\left(Z_{i j}^{3}\right)^{Z_{j j^{\prime}}^{-1}}$.

As a result, we get a factorized expression, which, by [8], determines the diffeomorphism type of our surface, and, by Van Kampen [20], determines $\pi_{1}\left(\mathbb{C} \mathbb{P}^{2}-\bar{S}\right)$. This is explained in the following paragraphs.

Assume that we have a curve $\bar{S}$ in $\mathbb{C P}^{2}$ and its BMF. Then we can calculate the groups $\pi_{1}\left(\mathbb{C P}^{2}-\bar{S}\right)$ and $\pi_{1}\left(\mathbb{C}^{2}-S\right)\left(\right.$ where $\left.S=\bar{S} \cap \mathbb{C}^{2}\right)$.

Recall that a $g$-base is an ordered free base of $\pi(D \backslash F, v)$, where $D$ is a closed disc, $F$ is a finite set in $\operatorname{Int}(D), v \in \partial D$ which satisfies several conditions; see [14; 15] for the explicit definition.

Let $\left\{\Gamma_{i}\right\}$ be a $g$-base of $G=\pi_{1}\left(\mathbb{C}_{u}-S, u\right)$, where $\mathbb{C}_{u}=\mathbb{C} \times u$, and here $S=\mathbb{C}_{u} \cap S$. We cite now the Zariski-Van Kampen Theorem (for cuspidal curves) in order to compute the relations between the generators in $G$. 
Theorem 2.5 (Zariski-Van Kampen - cuspidal curves version) Let $\bar{S}$ be a cuspidal curve in $\mathbb{C P}^{2}$. Let $S=\mathbb{C}^{2} \cap \bar{S}$. Let $\varphi$ be a braid monodromy factorization w.r.t. $S$ and $u$. Let $\varphi=\prod_{j=1}^{p} V_{j}^{v_{j}}$, where $V_{j}$ is a half-twist and $v_{j}=1,2,3$.

For every $j=1, \ldots, p$, let $A_{j}, B_{j} \in \pi_{1}\left(\mathbb{C}_{u}-S, u\right)$ be such that $A_{j}, B_{j}$ can be extended to a $g$-base of $\pi_{1}\left(\mathbb{C}_{u}-S, u\right)$ and $\left(A_{j}\right) V_{j}=B_{j}$. Let $\left\{\Gamma_{i}\right\}$ be a $g$-base of $\pi_{1}\left(\mathbb{C}_{u}-S, u\right)$ corresponding to the $\left\{A_{i}, B_{i}\right\}$, where $A_{i}, B_{i}$ are expressed in terms of $\Gamma_{i}$. Then $\pi_{1}\left(\mathbb{C}^{2}-S, u\right)$ is generated by the images of $\left\{\Gamma_{i}\right\}$ in $\pi_{1}\left(\mathbb{C}^{2}-S, u\right)$ and the only relations are those implied from $\left\{V_{j}^{v_{j}}\right\}$, as follows:

$$
\begin{cases}A_{j} \cdot B_{j}^{-1} & \text { if } v_{j}=1 \\ {\left[A_{j}, B_{j}\right]=1} & \text { if } v_{j}=2 \\ \left\langle A_{j}, B_{j}\right\rangle=1 & \text { if } v_{j}=3 .\end{cases}
$$

$\pi_{1}\left(\mathbb{C} \mathbb{P}^{2}-\bar{S}, *\right)$ is generated by $\left\{\Gamma_{i}\right\}$ with the above relations and one more relation $\prod_{i} \Gamma_{i}=1$.

Figure 8 illustrates how to find $A_{i}, B_{i}$ from the half-twist $V_{i}=H(\sigma)$.
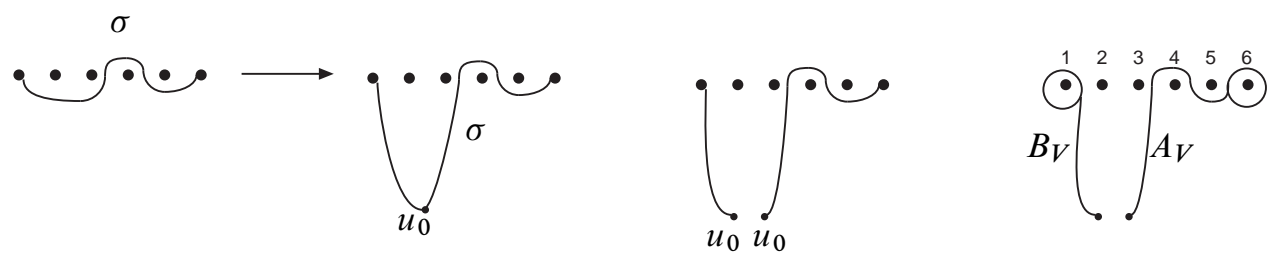

Figure 8

So:

$$
A_{V}=\Gamma_{4}^{-1} \Gamma_{6} \Gamma_{4}, B_{V}=\Gamma_{1} .
$$

We finish this subsection by recalling the definition of $\widetilde{B}_{n}$.

\section{Definition}

(i) Let $X, Y$ be two half-twists in $B_{n}=B_{n}(D, K)$. We say that $X, Y$ are transversal if they are defined by two simple paths $\xi, \eta$ which intersect transversally in one point different from their ends.

(ii) Let $N$ be the normal subgroup of $B_{n}$ generated by conjugates of $[X, Y]$, where $X, Y$ is a transversal pair of half-twists. Define $\widetilde{B}_{n}=B_{n} / N$. 
(iii) Let $Y_{i}, i=1, \ldots, 4$ be four half-twists in $B_{n}$ (resp. $\widetilde{B}_{n}$ ) corresponding to simple paths $\eta_{1}, \ldots, \eta_{4}$. Assume that $\eta_{i}, i=1, \ldots, 4$, could be chosen so that they form a quadrangle without self intersections and such that in its interior there are no points of $K$. Then we say that $Y_{1}, Y_{2}, Y_{3}, Y_{4}$ form a good quadrangle in $B_{n}$ (resp. in $\left.\widetilde{B}_{n}\right)$.

Lemma 2.6 If $y_{1}, y_{2}, y_{3}, y_{4} \in \widetilde{B}_{n}$ form a good quadrangle then $y_{1}^{2} y_{3}^{2}=y_{2}^{2} y_{4}^{2}$.

Proof See Moishezon [11, section 1.1].

\section{Computing the BMFs}

Let $\varphi_{1}, \varphi_{2}$ be the BMF of the branch curve of the first (resp. second) K3 surface. Before computing $\varphi_{1}, \varphi_{2}$, we need some notation. Denote the intersection lines on $\left(X_{i}\right)_{0}$ as $\left\{\hat{L}_{i, j}\right\}_{j=1}^{24}, i=1,2$ (recall that $\left(X_{i}\right)_{0}$ is the degeneration of the K3surfaces $\left.X_{i}, i=1,2\right)$, and by $\left\{\hat{v}_{i, j}\right\}_{j=1}^{10}, i=1,2$ the intersection points of these lines. Take generic projections $\pi_{i}:\left(X_{i}\right)_{0} \rightarrow \mathbb{C P}^{2}$, and let $\left(S_{i}\right)_{0}$ be the branch curve in $\mathbb{C P}^{2},\left(\gamma_{i}\right)_{0}$ - their braid monodromy, and $L_{i, j}=\pi_{i}\left(\hat{L}_{i, j}\right), i=1,2, j, \ldots, 24$. So, $\left(S_{i}\right)_{0}=\bigcup_{j=1}^{24} L_{i, j}, v_{i, j} \doteq \pi_{i}\left(\hat{v}_{i, j}\right), i=1,2, j=1, \ldots, 24$ are the singular points of $\left(S_{i}\right)_{0}$. Let $C_{i}$ be the union of all lines connecting pairs of the $v_{i, j} \in\left(S_{i}\right)_{0}$. $\left(S_{i}\right)_{0}$ is a subcurve of $C_{i}$. By [14, Theorem IX], we get a full description of the braid monodromy of $C_{i}: \Delta_{C_{i}}^{2}=\Pi_{j=10}^{1} C_{i, j} \Delta_{v_{i, j}}^{2}(i=1,2)$ with an appropriate description of the corresponding braids. We use this formula to obtain a description of $\left(\varphi_{i}\right)_{0}$ by deleting factors that involve lines which do not appear in $\left(S_{i}\right)_{0}$. Thus, we get $\left(\gamma_{i}\right)_{0}=\Delta_{\left(S_{i}\right)_{0}}^{2}=\Pi_{j=10}^{1} \widetilde{C}_{i, j} \widetilde{\Delta}_{v_{i, j}}^{2}$. We describe each factor separately.

$\widetilde{C}_{i, j}:$ The factors $\widetilde{C}_{i, j}$ correspond to parasitic intersections; these are intersections created by lines that do not intersect in $\mathbb{C} \mathbb{P}^{9}$ but may intersect in $\mathbb{C P}^{2}$. By [14] we know that $\widetilde{C}_{i, j}=\prod_{v_{i, j} \in L_{i, t}} D_{i, t}$, where $D_{i, t}=\prod_{\substack{p<t \\ L_{i, p} \cap L_{i, t}=\varnothing}} \widetilde{Z}_{p t}^{2}$. For $i=1$, the global BMF, together with the $\widetilde{C}_{1, j}$ is presented in Amram-CilibertoMiranda-Teicher [1, Section 4.1]. For $i=2$, we have (by [14, Theorem X.2.1]):

$$
\begin{aligned}
D_{2,1}=\text { Id } & D_{2,2}=\underline{Z}_{1,2}^{2} & D_{2,3} & =\underline{Z}_{2,3}^{2} \\
& (2) & & (2-3)(4) \\
D_{2,4}=\underline{Z}_{1,4}^{2} & D_{2,5}=\bar{Z}_{2,5}^{2} \bar{Z}_{3,5}^{2} \underline{Z}_{4,5}^{2} & D_{2,6} & =\underline{Z}_{1,6}^{2} \underline{Z}_{3,6}^{2} \underline{Z}_{4,6}^{2}
\end{aligned}
$$




$$
\begin{aligned}
& D_{2,7}=\prod_{i=2,3,4,6} \bar{Z}_{i, 7}^{2} \quad D_{2,8}=\prod_{i=1,3,4,5} \bar{Z}_{i, 8}^{2} \quad D_{2,9}=\bar{Z}_{2,9}^{2} \underline{Z}_{4,9}^{(5-6)} \underline{Z}_{5,9}^{(6)} \underline{Z}_{6,9}^{2} \\
& D_{2,10}=\prod_{i=1,2,3,4} \bar{Z}_{i, 10}^{2} \quad D_{2,11}=\prod_{\substack{i=2,3,4, 6,8,9,10}} \bar{Z}_{i, 11}^{2} \quad D_{2,12}=\prod_{\substack{i=1,3,4,5 \\
7,9,10}} \bar{Z}_{i, 12}^{2} \\
& D_{2,13}=\prod_{\substack{i=1,3,5,(11-12) \\
7, \ldots, 10}} \bar{Z}_{i, 13}^{2} \quad D_{2,14}=\prod_{\substack{i=1, \ldots, 4 \\
7,8,9}} \bar{Z}_{i, 14}^{2} \quad D_{2,15}=\prod_{\substack{i=2, \ldots, 14 \\
1 \neq 5,7,11}} \bar{Z}_{i, 15}^{2} \\
& D_{2,16}=\prod_{\substack{i=2, \ldots, 14 \\
i \neq 3,9}} \bar{Z}_{i, 16}^{2} \quad D_{2,17}=\prod_{\substack{i=1, \ldots, 14 \\
i \neq 2,4,13}} \bar{Z}_{i, 17}^{2}(15-16) \quad D_{2,18}=\prod_{\substack{i=1, \ldots, 14 \\
i \neq 3,4}} \bar{Z}_{i, 18}^{2} \\
& D_{2,19}=\prod_{i=1, \ldots, 10} \bar{Z}_{i, 19}^{2} \quad D_{2,20}=\prod_{\substack{i=2, \ldots, 19 \\
i \neq 2,6,8,12}} \bar{Z}_{i, 20}^{2} \quad D_{2,21}=\prod_{\substack{i=2, \ldots, 19 \\
i \neq 3,9,16}} \bar{Z}_{i, 21}^{2}
\end{aligned}
$$

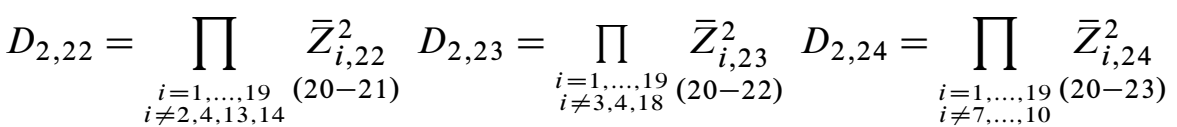

and

$$
\begin{aligned}
& \widetilde{C}_{2,1}=\prod_{\substack{t=5,7 \\
11,15}} D_{2, t} \quad \widetilde{C}_{2,2}=\prod_{\substack{t=2,6,8 \\
12,20}} D_{2, t} \widetilde{C}_{2,3}=\prod_{\substack{t=3,9 \\
16,21}} D_{2, t} \\
& \widetilde{C}_{2,4}=\prod_{\substack{t=4,13 \\
17,22}} D_{2, t} \quad \widetilde{C}_{2,5}=\prod_{t=18,23} D_{2, t} \quad \widetilde{C}_{2,6}=\prod_{t=10,14} D_{2, t} \\
& \widetilde{C}_{2,7}=D_{2,24} \quad \widetilde{C}_{2,18}=D_{2,19} \quad \widetilde{C}_{2,9}=\widetilde{C}_{2,10}=\mathrm{Id} .
\end{aligned}
$$

Recall that a point in a totally degenerated surface is called a $k$-point if it is a singular point which is the intersection of $k$ planes.

$\widetilde{\Delta}_{v_{i, j}}^{2}$ : In $\left(S_{1}\right)_{0}$, we have six points, which are 6-point $\left(v_{1, j}, j=2,4,5,7,9,10\right)$ and four points which are 3-point $\left(v_{1, j}, j=1,3,6,8\right.$; note that the regeneration of this 3-point is not similar to the regular 3-point. See [1] for the braid monodromy factorization of the regeneration of our 3-point.

In $\left(S_{2}\right)_{0}$, we have eight points which are 5-point $\left(v_{2, j}, 1 \leq j \leq 10, j \neq 5,6\right)$ and two points which are 4-point $\left(v_{2, j}, j=5,6\right)$. Note that the original branch curve, $S_{2}$, has also a few extra branch points. The existence of the extra branch points will be proved later (see Proposition 3.7).

The local braid monodromies, which are $\widetilde{\Delta}_{v_{2, j}}^{2}$, are introduced and regenerated in the following paragraphs. We denote the outcoming local BMF, resulting from the total regeneration $\widetilde{\Delta}_{v_{2, j}}^{2}$, as $\varphi_{2, j}$. Thus after performing a total regeneration 
to the whole BMF, the resulting BMF will be of the form $\varphi_{2}=\prod_{i=10}^{1} C_{i} \varphi_{i} \prod b_{i}$, where $b_{i}$ are braids corresponding to the extra branch points.

Before presenting the expressions for local and global BMFs, we give some

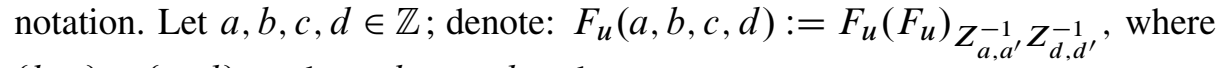
$\{b, c\}<\{a, d\}$, and $c<b, a<d$ and

$$
\begin{gathered}
F_{u}=Z_{b b^{\prime}, a}^{(3)} Z_{a^{\prime} d}^{2}\left(Z_{a d}^{2}\right)_{Z_{b b^{\prime} a}^{2}}\left(Z_{b b^{\prime}, d}^{3}\right)_{Z_{b b^{\prime}, a}^{2}}\left(Z_{c b^{\prime}}\right)_{Z_{b^{\prime} d} Z_{b^{\prime} a}^{2}} \\
\left(Z_{c^{\prime} b}\right)_{Z_{b d} Z_{b a}^{2} Z_{b b^{\prime}}^{2}} \\
F_{m}(a, b, c, d):=F_{m} \cdot\left(F_{m}\right)_{Z_{a, a^{\prime}}^{-1} Z_{d, d^{\prime}}^{-1} \text { where } a<\{b, c\}<d \text { and }} \\
F_{m}=Z_{a^{\prime}, c c^{\prime}}^{(3)} \cdot Z_{b b^{\prime}, d}^{(3)} \cdot \widetilde{Z}_{c, b^{\prime}} \cdot \widetilde{Z}_{b^{\prime}, c}\left(\underline{Z}_{a^{\prime}, d}^{2}\right)_{Z_{c^{\prime}, c c^{\prime}}^{2}} \cdot \underline{Z}_{a d}^{2}
\end{gathered}
$$

where

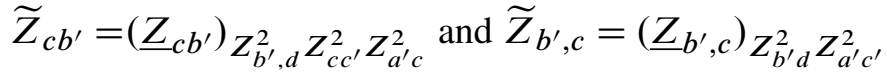

$$
\begin{aligned}
& F_{\ell}(a, b, c, d):=F_{\ell} \cdot\left(F_{\ell}\right)_{Z_{a, a^{\prime}}^{-1} Z_{d, d^{\prime}}^{-1}} \text { where }\{b, c\}>\{a, b\} \text { and } \\
& F_{\ell}=Z_{a^{\prime} d}^{2} \cdot Z_{d^{\prime}, c c^{\prime}}^{(3)}\left(Z_{a^{\prime} d^{\prime}}^{2}\right)_{Z_{d^{\prime}, c c^{\prime}}^{2}} Z_{a^{\prime} d}^{2}\left(Z_{a^{\prime}, c c^{\prime}}^{(3)} Z_{a^{\prime} d}^{2}\right.
\end{aligned}
$$

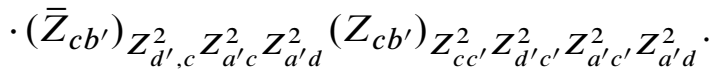

Note that for $\left(\varphi_{1}\right)_{0}$ and the singular points of $\left(S_{1}\right)_{0}$, the regeneration process was already done [1], and thus we have the following theorem.

Theorem 3.1 The BMF of the branch curve of $X_{1}$ is

$$
\varphi_{1}=\prod_{j=10}^{1} C_{1, j} \varphi_{1, j}
$$

where $C_{1, j}, \varphi_{1, j}$ can be found in [1].

Proof See [1].

Thus, we have to compute the BMF of the branch curve of $X_{2}$. We begin by citing the results about the points $v_{2,5}$ and $v_{2,6}$; these are 4-points and for this type, the BMF of a fully regenerated neighbourhood was computed in Amram-Teicher [3].

Proposition 3.2 The local braid monodromy $\varphi_{2,5}$ in a small neighbourhood around $v_{2,5}$ has the following form:

$$
\varphi_{2,5}=F_{u}(18,4,3,23)
$$


and the local braid monodromy $\varphi_{2,6}$ (for $v_{2,6}$ ) has the same form, when substituting $3 \rightarrow 5,4 \rightarrow 6,18 \rightarrow 10,14 \rightarrow 23$.

Proof See [3].

We now move on to compute the local braid monodromy around a small neighbourhood of $v_{2,3}$, which is a 5-point. We will give - for this point - a detailed treatment for the computation of the local BMF, while for the other points $\left(v_{2, j}, j=1,2,4,7, \ldots, 10\right)$ we will just give the final results.

We examine the point $v_{2,3}$ in the degenerated surface $\left(X_{2}\right)_{0}$. Drawing a local neighbourhood of $v_{2,3}$ and numerating the lines $-L_{i}(1 \leq i \leq 5)$ locally, we get Figure 9.

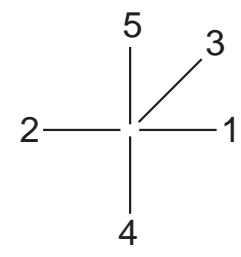

Figure 9

By the degeneration process, line 3 is regenerated first. By the claim in MoishezonTeicher [16, Section 2], we know that line $L_{3}$ is regenerated into a conic. More explicitly, we get that after regenerating $V=\bigcup_{i=1}^{5} L_{i}$ in a small neighbourhood $U$ of $v_{2,3}, L_{3}$ turns into a conic $Q_{3}$ such that $Q_{3}$ is tangent to $L_{1}$ and $L_{5}$. Denote the resulting branch curve, after the regeneration by $\widetilde{V}$. Thus, the singularities of $T=\widetilde{V} \cap U$ are as in Figure 10 .

Proposition 3.3 The local braid monodromy factorization of the above configuration is

$$
\widetilde{\varphi}=Z_{2,3}^{2} Z_{3^{\prime}, 4}^{2} Z_{1,3}^{4} \bar{Z}_{3^{\prime}, 5}^{4} \widetilde{Z}_{3^{\prime}, 4}^{2} \widetilde{Z}_{2,3}^{2} \widetilde{Z}_{3,3^{\prime}}^{2}\left(\Delta^{2}\langle 1,2,4,5\rangle\right)^{Z_{3,4}^{-2}}
$$

where the braids $\widetilde{Z}_{3^{\prime}, 4}, \widetilde{Z}_{2,3}, \widetilde{Z}_{3,3^{\prime}}$ correspond to the paths shown in Figure 11 .

Proof Let $\left\{p_{j}\right\}_{j=1}^{8}$ be the singular points of a small neighbourhood (that is $U$ ) of $v_{2,3}$ (see Figure 10) with respect to $\pi_{1}$ (the projection to the $X$-axis) as follows.

(i) $\left\{p_{1}, p_{2}\right\},\left\{p_{2}, p_{5}\right\}$ - the intersection points of $Q_{3}$ with $L_{2}, L_{4}$. 


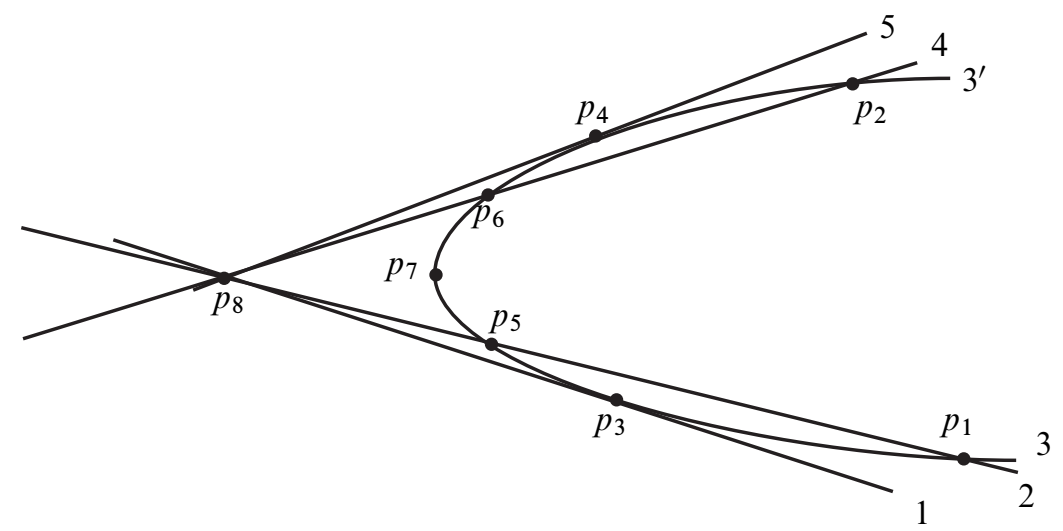

Figure 10

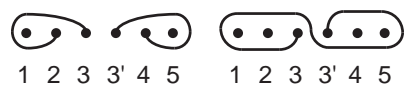

Figure 11

(ii) $p_{3}, p_{4}$ - the tangent points of $Q_{3}$ and $L_{1}, L_{5}$.

(iii) $\quad p_{7}-$ the branch point of $Q_{3}$.

(iv) $p_{8}-$ the intersection point of $\left\{L_{i}\right\}_{i=1,2,4,5}$.

Let $E$ (resp. $D)$ be a closed disk on the $X$-axis (resp. $Y$-axis). Let $N=\left\{x\left(p_{j}\right)=\right.$ $\left.x_{j} \mid 1 \leq j \leq 8\right\}$, s.t. $N \subset E-\partial E$. Let $M$ be a real point on the $x$-axis, s.t. $x_{j} \ll$ $M, \forall x_{j} \in N, 1 \leq j \leq 8$. There is a $g$-base $\ell\left(\gamma_{j}\right)_{j=1}^{8}$ of $\pi_{1}(E-N, u)$, s.t. each path $\gamma_{j}$ is below the real line and the values of $\varphi_{M}$ with respect to this base and $E \times D$ are the ones given in the proposition. We look for $\varphi_{M}\left(\ell\left(\gamma_{j}\right)\right)$ for $j=1, \ldots, 8$. Choose a $g$-base $\ell\left(\gamma_{j}\right)_{j=1}^{8}$ as above and put all the data in the following table. 


\begin{tabular}{c|c|c|c}
$j$ & $\lambda_{j}$ & $\varepsilon_{j}$ & $\delta_{j}$ \\
\hline 1 & $\langle 2,3\rangle$ & 2 & $\Delta\langle 2,3\rangle$ \\
2 & $\left\langle 3^{\prime}, 4\right\rangle$ & 2 & $\Delta\left\langle 3^{\prime}, 4\right\rangle$ \\
3 & $\langle 1,2\rangle$ & 4 & $\Delta^{2}\langle 1,2\rangle$ \\
4 & $\langle 4,5\rangle$ & 4 & $\Delta^{2}\langle 4,5\rangle$ \\
5 & $\left\langle 3^{\prime}, 4\right\rangle$ & 2 & $\Delta\left\langle 3^{\prime}, 4\right\rangle$ \\
6 & $\langle 2,3\rangle$ & 2 & $\Delta\langle 2,3\rangle$ \\
7 & $\left\langle 3,3^{\prime}\right\rangle$ & 1 & $\Delta_{I R}^{1 / 2}\langle 2\rangle$ \\
8 & $\langle 1,2,4,5\rangle$ & 2 & -
\end{tabular}

So, we get the following:

$$
\begin{aligned}
\xi_{x_{1}} & =z_{2,3} \\
\varphi_{M}\left(\ell\left(\gamma_{1}\right)\right) & =Z_{2,3}^{2} \\
\xi_{x_{2}} & =z_{3^{\prime}, 4}
\end{aligned}
$$

$(\Delta\langle 2,3\rangle$ does not affect this path)

$$
\varphi_{M}\left(\ell\left(\gamma_{2}\right)\right)=Z_{3,4}^{2}
$$

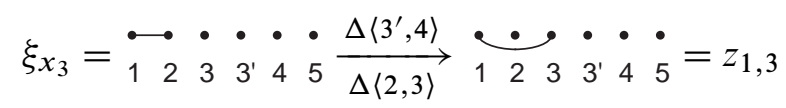

$\varphi_{M}\left(\ell\left(\gamma_{3}\right)\right)=Z_{1,3}^{4}$

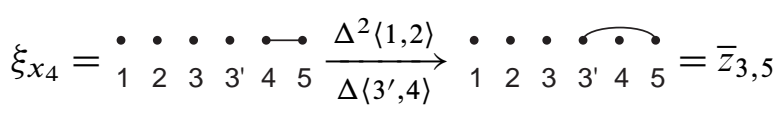

$(\Delta\langle 2,3\rangle$ does not affect this path

$$
\begin{aligned}
& \varphi_{M}\left(\ell\left(\gamma_{4}\right)\right)=\bar{Z}_{3^{\prime}, 5}^{4}
\end{aligned}
$$

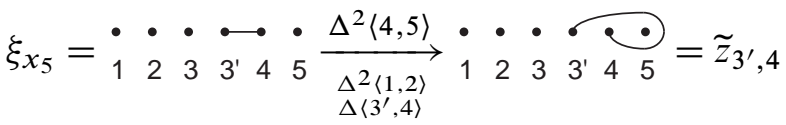

$(\Delta\langle 2,3\rangle$ does not affect this path)

$$
\begin{aligned}
& \varphi_{M}\left(\ell\left(\gamma_{5}\right)\right)=\widetilde{Z}_{3^{\prime}, 4}^{2}
\end{aligned}
$$

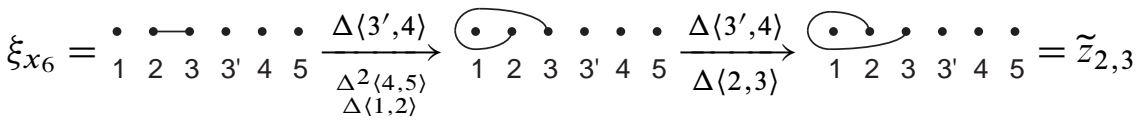

$$
\begin{aligned}
& \varphi_{M}\left(\ell\left(\gamma_{6}\right)\right)=\widetilde{Z}_{2,3}^{2}
\end{aligned}
$$




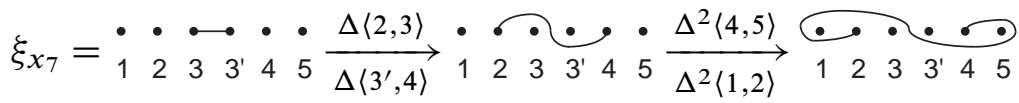

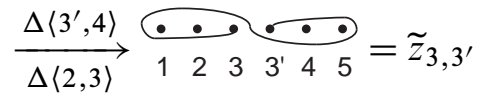

$$
\begin{aligned}
& \varphi_{M}\left(\ell\left(\gamma_{7}\right)\right)=\widetilde{Z}_{3,3^{\prime}}
\end{aligned}
$$

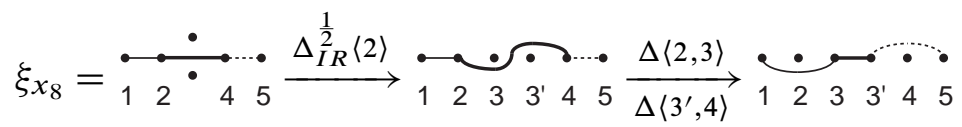

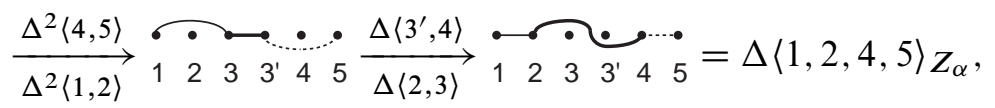

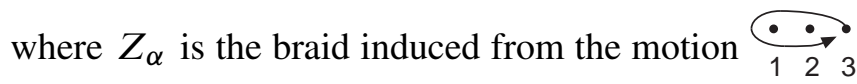

$$
\varphi_{M}\left(\ell\left(\gamma_{8}\right)\right)=\Delta^{2}\langle 1,2,4,5\rangle_{Z_{\alpha}}
$$

The following regeneration regenerates a small neighborhood of $\bigcup_{i=1,2,4,5} L_{i}$, which is, by definition, a 4-point. Since this type of 4-point and its BMF of its regeneration was treated earlier [3], we can find out what is the BMF of $v_{2,3}$ after the full regeneration.

Proposition 3.4 The local $B M F \varphi_{2,3}$ around a small neighborhood of $v_{2,3}$ is:

$$
\begin{aligned}
\varphi_{2,3}= & Z_{2^{\prime}, 3}^{2} Z_{2,3}^{2} Z_{3^{\prime}, 4^{\prime}}^{2} Z_{3^{\prime}, 4}^{2} Z_{11^{\prime}, 3}^{(3)} \cdot \bar{Z}_{3^{\prime}, 55^{\prime}}^{(3)} \widetilde{Z}_{3^{\prime}, 4^{\prime}}^{2} \widetilde{Z}_{3^{\prime}, 4}^{2} \widetilde{Z}_{2^{\prime}, 3}^{2} \widetilde{Z}_{2,3}^{2} \\
& \widetilde{Z}_{3,3^{\prime}}\left(F_{3} \cdot\left(F_{3}\right)_{\vartheta}\right)_{Z_{\alpha}}
\end{aligned}
$$

where $\vartheta=Z_{4,4^{\prime}}^{-1} \cdot Z_{5,5^{\prime}}^{-1}$, the braids $\widetilde{Z}_{3^{\prime}, 4^{\prime}}, \widetilde{Z}_{3^{\prime}, 4}, \widetilde{Z}_{2^{\prime}, 3}, \widetilde{Z}_{2,3}, \widetilde{Z}_{3,3^{\prime}}$ correspond to the following paths:

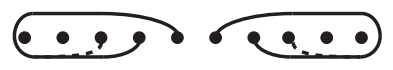

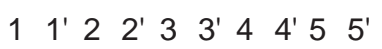

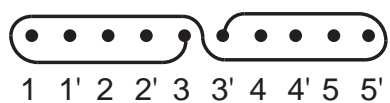

and $Z_{\alpha}$ is the braid induced from the motion shown in Figure 12 and

$$
F_{3}=Z_{22^{\prime}, 4}^{(3)} Z_{4^{\prime}, 5}^{2}\left(Z_{4,5}^{2}\right)_{Z_{22^{\prime}, 4}^{2}}^{2}\left(Z_{22^{\prime}, 5}^{(3)}\right)_{22^{\prime}, 4}^{2}\left(Z_{1,2^{\prime}}\right)_{Z_{2^{\prime}, 5}^{2} Z_{2^{\prime}, 4}^{2}}\left(Z_{1^{\prime}, 2}\right)_{Z_{2,5}}^{2} Z_{2,4}^{2} Z_{2,2^{\prime}}^{2} .
$$

Proof Using the regeneration rules, we replace

(i) $Z_{2,3}^{2}\left(Z_{3,4}^{2}, \tilde{Z}_{3,4}^{2}, \widetilde{Z}_{2,3}^{2}\right)$ by $Z_{22^{\prime}, 3}^{2} \quad$ (resp. $Z_{3,44^{\prime}}^{2}, \widetilde{Z}_{3,44^{\prime}}^{2}, \widetilde{Z}_{22^{\prime}, 3}^{2}$ ) (by the second regeneration rule) 


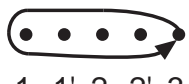

Figure 12

(ii) $Z_{1,3}^{4} \quad\left(\bar{Z}_{3^{\prime}, 5}^{4}\right)$ by $Z_{11^{\prime}, 3}^{(3)} \quad$ (resp. $\left.\bar{Z}_{3^{\prime}, 55^{\prime}}^{(3)}\right)$ (by the third regeneration rule)

(iii) $\Delta^{2}\langle 1,2,4,5\rangle$ by $F_{3} \cdot\left(F_{3}\right)_{\vartheta}$.

Remark 3.5 Note that the last BMF was given when numerating the lines in the neighbourhood of $v_{2,3}$ locally. So, when numerating globally, we get:

$$
\begin{aligned}
\varphi_{2,3}= & Z_{3^{\prime}, 9}^{2} Z_{3,9}^{2} Z_{9^{\prime}, 16^{\prime}}^{2} Z_{9^{\prime}, 16}^{2} Z_{11^{\prime}, 9}^{(3)} \bar{Z}_{9^{\prime}, 2121^{\prime}}^{(3)} \widetilde{Z}_{9^{\prime}, 16^{\prime}}^{2} \widetilde{Z}_{9^{\prime}, 16}^{2} \widetilde{Z}_{3^{\prime}, 9}^{2} \widetilde{Z}_{3,9}^{2} \\
& \widetilde{Z}_{9,9^{\prime}} \cdot\left(F_{3} \cdot\left(F_{3}\right)_{\vartheta}\right) Z_{\alpha_{3}}
\end{aligned}
$$

where $\vartheta=Z_{16,16^{\prime}}^{-1} Z_{21,21^{\prime}}^{-1}, Z_{\alpha_{3}}$ is the braid induced from the motion shown in Figure 13 and

$$
\begin{aligned}
F_{3}= & Z_{33^{\prime}, 16}^{(3)} Z_{16^{\prime}, 21}^{2}\left(Z_{16,21}^{2}\right)_{Z_{33^{\prime}, 16}^{2}}\left(Z_{33^{\prime}, 21}^{(3)}\right)_{Z_{33^{\prime}, 16}^{2}}\left(Z_{1,3^{\prime}}\right)_{3_{3^{\prime}, 21}^{2}} Z_{3^{\prime}, 16}^{2} \\
& \cdot\left(Z_{1^{\prime}, 3}\right)_{Z_{3,21}^{2}}^{2} Z_{3,16}^{2} Z_{3,3^{\prime}}^{2} .
\end{aligned}
$$

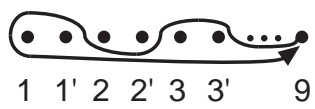

Figure 13

We now write the other BMFs.

Proposition 3.6 The local braid monodromy $\varphi_{2,1}$ is:

$\varphi_{2,1}=Z_{1111^{\prime}, 15}^{(3)}\left(F_{u}(11,5,1,7)\right)^{Z_{1111^{\prime}, 15}^{2}} \stackrel{\stackrel{\left(7-7^{\prime}\right.}{\left.5-5^{\prime}\right)}}{Z_{11^{\prime}, 15}} \widetilde{Z}_{15,15^{\prime}} Z_{55^{\prime}, 1515^{\prime}}^{\left(7-7^{\prime}\right)} \widetilde{Z}_{77^{\prime}, 15^{\prime}}^{2} Z_{77^{\prime}, 15}^{2}$

where $\widetilde{Z}_{15,15^{\prime}}, \widetilde{Z}_{77,15^{\prime}}$ correspond to the paths shown in Figure 14.

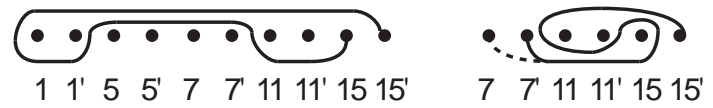

Figure 14

Algebraic 83 Geometric Topology, Volume 8 (2008) 
The local braid monodromy $\varphi_{2,2}$ is:

$$
\begin{aligned}
\varphi_{2,2}= & Z_{1212^{\prime}, 20}^{2} Z_{88^{\prime}, 20}^{(3)} \widetilde{Z}_{1212^{\prime}, 20}^{2}\left(F_{u}(8,6,2,12)\right)_{Z_{\alpha_{2}}} Z_{22,20^{\prime}}^{\left(6-6^{\prime}\right)} \widetilde{Z}_{20,20^{\prime}}^{2} \\
& \widetilde{Z}_{66^{\prime}, 20^{\prime}}^{2} Z_{66^{\prime}, 20}^{2}
\end{aligned}
$$

where $\widetilde{Z}_{1212^{\prime}, 20}, \widetilde{Z}_{20,20^{\prime}}, \widetilde{Z}_{66^{\prime}, 20^{\prime}}$ correspond to the paths shown in Figure 15 and

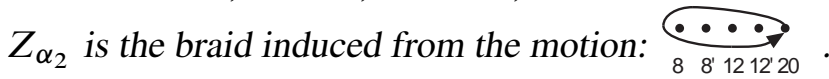

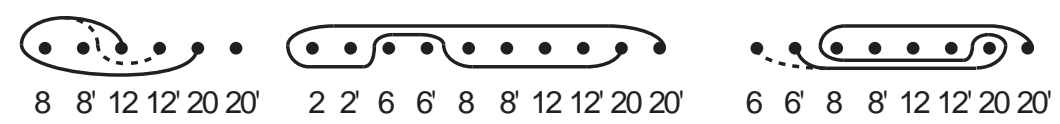

Figure 15

The local braid monodromy $\varphi_{2,4}$ is:

$$
\begin{aligned}
\varphi_{2,4}= & Z_{44^{\prime}, 13}^{2} Z_{13^{\prime}, 1717^{\prime}}^{(3)} Z_{22^{\prime}, 13}^{(3)} Z_{44^{\prime}, 13}^{2} \widetilde{Z}_{13,13^{\prime}} \underline{Z}_{13^{\prime}, 2222^{\prime}}^{\left(17-17^{\prime}\right)} \\
& \widetilde{Z}_{13,2222^{\prime}}^{2}\left(F_{u}(22,4,2,17)\right)_{\alpha_{4}}
\end{aligned}
$$

where $\widetilde{Z}_{13,13^{\prime}}, \widetilde{Z}_{13^{\prime}, 2222^{\prime}}$ correspond to the paths shown in Figure 16 and $Z_{\alpha_{4}}$ is the

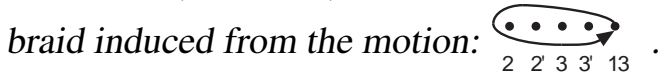

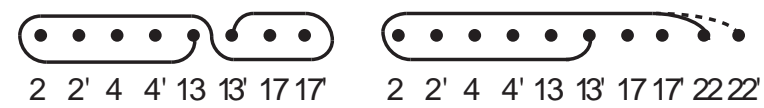

Figure 16

The local braid monodromy $\varphi_{2,7}$ is:

$\varphi_{2,7}=Z_{88^{\prime}, 9}^{2} Z_{1010^{\prime}, 9^{\prime}}^{2} Z_{77^{\prime}, 9}^{(3)} \underline{Z}_{9^{\prime}, 2424^{\prime}}^{\left(10-10^{\prime}\right)} \widetilde{Z}_{9^{\prime}, 1010^{\prime}}^{2} \widetilde{Z}_{88^{\prime}, 9}^{2} \widetilde{Z}_{9,9^{\prime}}\left(F_{\ell}(7,10,24,8)\right)_{\alpha_{7}}$

where $\widetilde{Z}_{9^{\prime}, 1010^{\prime}}, \widetilde{Z}_{88^{\prime}, 9^{\prime}}, \widetilde{Z}_{9,9^{\prime}}$ correspond to the paths shown in Figure 17 and $Z_{\alpha_{7}}$ is the braid induced from the motion: $\underset{77889}{\bullet \bullet ⿰}$.

The local braid monodromy $\varphi_{2,8}$ is:

$$
\begin{gathered}
\varphi_{2,8}=Z_{13^{\prime}, 1414^{\prime}}^{2} Z_{1212^{\prime}, 13}^{(3)} \stackrel{\left(14-14^{\prime}\right)}{(3)}{\underline{Z 13^{\prime}, 1919^{\prime}}}_{13^{\prime}, 1414^{\prime}}^{(} \widetilde{Z}_{13,13^{\prime}} \widetilde{Z}_{1111^{\prime}, 13^{\prime}}^{2} \\
\left.Z_{1111^{\prime}, 13}^{2} F_{\ell}(12,14,19,11)\right)_{Z_{\alpha_{8}}}
\end{gathered}
$$

Algebraic $8 \mathcal{G}$ Geometric Topology, Volume 8 (2008) 

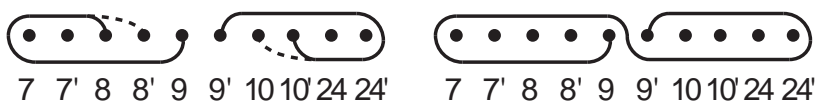

Figure 17

where $\widetilde{Z}_{13^{\prime}, 1414^{\prime}}, \widetilde{Z}_{1111^{\prime}, 13^{\prime}}, \widetilde{Z}_{13,13^{\prime}}$ correspond to the paths shown in Figure 18 and $Z_{\alpha_{8}}$ is the braid induced from the motion: $\underset{1111^{121213}}{\stackrel{\bullet}{\bullet}}$.

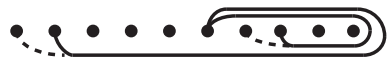

$1111^{\prime} 1212^{\prime} 1313^{\prime} 1414^{\prime} 19$ 19'

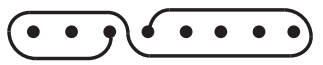

$1212^{\prime} 1313^{\prime} 1414^{\prime} 19$ 19'

Figure 18

The local braid monodromy $\varphi_{2,9}$ is:

$$
\begin{aligned}
\varphi_{2,9}= & \left.Z_{15^{\prime}, 1616^{\prime}}^{(3)} F_{\ell}(17,19,18,16)\right)_{Z_{15^{\prime}, 1616^{\prime}}^{2}} \underline{Z}_{15^{\prime}, 1919^{\prime}}^{\left(16-16^{\prime}\right)} \widetilde{Z}_{1515^{\prime}} \\
& \widetilde{Z}_{15,1818^{\prime}}^{2}{ }_{\left(16-16^{\prime}\right)}^{2} Z_{15^{\prime}, 1818^{\prime}} \bar{Z}_{15^{\prime}, 1717^{\prime}}^{2} \widetilde{Z}_{15,1717^{\prime}}^{2}
\end{aligned}
$$

where $\widetilde{Z}_{1515^{\prime}}, \widetilde{Z}_{151818^{\prime}}, \widetilde{Z}_{15,1717^{\prime}}$ correspond to the paths shown in Figure 19.

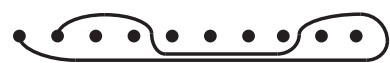

$1515^{\prime} 1616^{\prime} 17171818^{\prime} 1919^{\prime}$

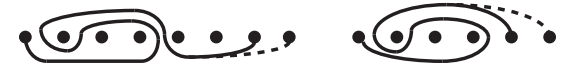

$1515^{\prime} 1616^{\prime} 17171818^{\prime} \quad 1515^{\prime} 1616^{\prime} 1717$

Figure 19

The local braid monodromy $\varphi_{2,10}$ is:

$$
\begin{gathered}
\varphi_{2,10}=Z_{20^{\prime}, 2121^{\prime}}^{2} \bar{Z}_{20^{\prime}, 2222^{\prime}}^{(3)} \widetilde{Z}_{20^{\prime}, 2121^{\prime}}^{2}\left(F_{\ell}(21,24,23,22)\right)_{\substack{Z_{10}\left(23-23^{\prime}\right) \\
Z_{20^{\prime}, 2424^{\prime}}^{(3)}}} \\
\widetilde{Z}_{2020^{\prime}} \bar{Z}_{20^{\prime}, 2323^{\prime}}^{2} \widetilde{Z}_{20,2323^{\prime}}^{2}
\end{gathered}
$$

where $\widetilde{Z}_{20^{\prime}, 2121^{\prime}}, \widetilde{Z}_{2020^{\prime}}, \widetilde{Z}_{20,2323^{\prime}}$ correspond to the paths shown in Figure 20 and

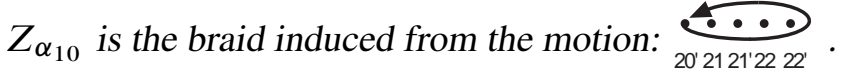

Performing the regeneration affects also the braids induced from the parasitic line intersection. Denote by $C_{2, i}$ the braid, which is created from $\widetilde{C}_{2, i}$ during the regeneration process. 


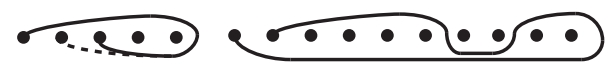

२०'21 21'22२2' 20 20'21 21'22२2' 2323'24 24'

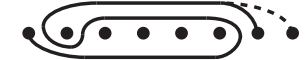

2020 '21 21'2222' 23

Figure 20

Every $\widetilde{C}_{2, i}$ is a product of a 2 -degree braid $Z_{i, j}^{2}$, which becomes, as a consequence of the second regeneration rule, an 8-degree braid: $Z_{i i^{\prime}, j j^{\prime}}^{2}=Z_{i^{\prime}, j^{\prime}}^{2} Z_{i^{\prime}, j}^{2} Z_{i, j^{\prime}}^{2} Z_{i, j}^{2}$. If the path representing the braid $Z_{i, j}^{2}$ was above/below a point $p$, then the induced braids would be above/below the points $p$ and $p^{\prime}$.

Before we present the global BMF, we have to check if there are extra branch points in the branch curves, that are created during the regeneration of a line $L_{i}$. An extra branch point contributes to a factorization the factor $Z_{i, i^{\prime}}$. (By "contributes" we mean that one should multiply the old factorization $Z_{i, i^{\prime}}$ from the right).

$X_{1}$ It was proven in [1, prop. 16] that the factorization $\varphi_{1}=\prod_{j=10}^{1} C_{1, j} \varphi_{1, j}$ is a BMF of the branch curve of $X_{1}$. Thus, there are no missing braids in the factorization above, and therefore there are no extra branch points.

$X_{2}$ Denote by $\widetilde{\Delta}=\prod_{j=10}^{1} C_{2, j} \varphi_{2, j}$. If $\widetilde{\Delta}$ was the BMF of the branch curve of $X_{2}$, then $\operatorname{deg}(\widetilde{\Delta})=\operatorname{deg}\left(\Delta_{48}^{2}\right)=48 \cdot(48-1)=2256$. We show that this is not the situation here. $\operatorname{deg}(\widetilde{\Delta})=\sum_{j=1}^{10} \operatorname{deg}\left(C_{2, j}\right)+\sum_{j=1}^{10} \operatorname{deg}\left(\varphi_{2, j}\right) \cdot \sum \operatorname{deg}\left(C_{2, j}\right)=$ $8 \cdot 184=1472$. For $j=5,6, v_{2, j}$ are 4-point, and by [16], $\operatorname{deg}\left(\varphi_{2,5}\right)=$ $\operatorname{deg}\left(\varphi_{2,6}\right)=48$. For $1 \leq j \leq 10, j \neq 5,6 v_{2, j}$ are 5-point. Although these points have different configurations, their BMFs $-\varphi_{2, j}$ still have 6 factors of degree 3, 8 factors of degree 2, one factor of degree 1 , and a factor representing the BMF of the regeneration of a 4-point, whose degree is 48 . Thus $\forall 1 \leq j \leq 10$, $j \neq 5,6, \operatorname{deg}\left(\varphi_{2, j}\right)=6 \cdot 3+8 \cdot 2+1+48=83$. So, $\operatorname{deg}(\widetilde{\Delta})=1472+2 \cdot 48+8 \cdot 83=$ $2232<2256$.

Define the forgetting homomorphisms:

$$
1 \leq i \leq 24 f_{i}: B_{48}\left[D,\left\{1,1^{\prime}, \ldots, 24,24^{\prime}\right\}\right] \rightarrow B_{2}\left[D,\left\{i, i^{\prime}\right\}\right]
$$

It is clear that if $\widetilde{\Delta}$ was a BMF, then $\forall i, \operatorname{deg}\left(f_{i}(\widetilde{\Delta})\right)=2$. However, this is not the case in the current situation. It was proven in Robb [18] (see also [19]), that if 
$\operatorname{deg}\left(f_{i}(\widetilde{\Delta})\right)=k<2$, then there are $(2-k)$ extra branch points, and so there is a contribution of the factorization $\prod_{m=1}^{2-k} Z_{i, i^{\prime}}$ to $\widetilde{\Delta}$.

\section{Proposition 3.7}

(i) The regeneration of the lines $L_{2, j}, j=3,4,5,6,10,14,18,23$ contributes the factors $Z_{j, j^{\prime}} \cdot Z_{j, j^{\prime}}$ to $\widetilde{\Delta}$.

(ii) The regeneration of the lines $L_{2, j} j=7,8,11,12,16,17,21,22$ contributes the factor $Z_{j, j^{\prime}}$ to $\widetilde{\Delta}$.

\section{Proof}

(i) We prove this case for $j=3$; the other cases are done using the same method. By Lemma 3.3.3 (or Proposition 3.3.4) in [19], it is enough to prove that $\operatorname{deg}\left(f_{3}(\widetilde{\Delta})\right)=0$. The braids coming from the parasitic intersection are sent by $f_{3}$ (and by any $f_{i}$, in fact) to Id, so it is enough to look only at the factors $\varphi_{2, k}, 1 \leq k \leq 10$ that involve braids, one of whose end points are 3 or $3^{\prime}$. The only suitable $k^{\prime} s$ are $k=5$ and $k=3$. Since $v_{2,3}$ and $v_{2,5}$ are both of 4-point, by Moishezon-Teicher [16, Lemma 8, (iv)], $\operatorname{deg}\left(f_{3}\left(\varphi_{2,3}\right)=\operatorname{deg}\left(f_{3}\left(\varphi_{2,5}\right)\right)=0\right.$. Therefore $\operatorname{deg}\left(f_{3}(\widetilde{\Delta})\right)=0$.

(ii) We prove for $j=7$; the other cases are done using the same method. It is enough to prove that $\operatorname{deg}\left(f_{7}(\widetilde{\Delta})\right)=1$ (by [19]).

As in (i), we only consider the factors $\varphi_{2,1}$ and $\varphi_{2,7} \cdot v_{2,1}$ is a 5-point. The first regeneration is of the line $L_{2,15}$, (which turns into a conic, that intersects the line $L_{2,7}$ at two nodes, which induce braids of the form $Z_{7,15}^{2}$ and $Z_{7,15^{\prime}}^{2}$ ), which does not contribute to the regeneration factors of the form $Z_{7,7^{\prime}}$. After this regeneration, we are left with the regeneration of a 4 -point, and by [16, Lemma 8 , (iv)], we get $\operatorname{deg}\left(f_{7}\left(\varphi_{2,1}\right)\right)=0$.

$v_{2,7}$ is also a 5-point. The first regeneration is of the line $L_{2,9}$, which turns into

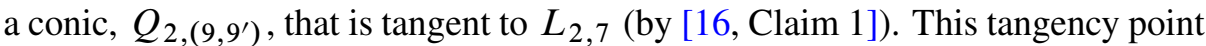
is regenerated into three cusps (see Moishezon-Teicher [15]) which induces the product of three braids $-Z_{7^{\prime}, 9}^{3} \cdot Z_{7,9}^{3} \cdot\left(Z_{7^{\prime}, 9}^{3}\right)_{Z_{7,7^{\prime}}^{-1}}=: Z_{77^{\prime}, 9}^{(3)}$. By [16, Lemma 2 , (i)], we see that $\operatorname{deg}\left(f_{7}\left(Z_{77^{\prime}, 9}^{(3)}\right)\right)=1$. Again, the regeneration afterwards of the 4-point does not contribute a factor of the form $Z_{7,7^{\prime}}$ to the factorization. Thus, we get $\operatorname{deg}\left(f_{7}\left(\varphi_{2,7}\right)\right)=1$, and $\operatorname{deg}\left(f_{7}(\widetilde{\Delta})\right)=1$. 
Define an ordered set

$$
\left\{i_{n}\right\}_{n=1}^{16}:=\{3,4,5,6,7,8,10,11,12,14,16,17,18,21,22,23\},
$$

and for $1 \leq n \leq 16$ let:

$$
b_{n}= \begin{cases}Z_{i_{n}, i_{n}^{\prime}} \cdot Z_{i_{n}, i_{n}^{\prime}} & i_{n}=\{3,4,5,6,10,14,18,23\} \\ Z_{i_{n}, i_{n}^{\prime}} & i_{n}=\{7,8,11,12,16,17,21,22\} .\end{cases}
$$

\section{Proposition 3.8}

$$
\varphi_{2}=\prod_{j=10}^{1} C_{2, j} \varphi_{2, j} \prod_{n=1}^{16} b_{n}
$$

is a braid monodromy factorization for $S_{2}$.

The proof is divided into a number of lemmas.

Lemma 3.9 $\varphi_{2}=\prod_{j=10}^{1} C_{2, j} \widetilde{\varphi}_{2, j} \prod_{n=1}^{16} b_{n}$ is a braid monodromy factorization for $S_{2}$, where $\widetilde{\varphi}_{2, j}=\left(\varphi_{2, j}\right)_{h_{j}}$ for some $h_{j} \in\left\langle Z_{k k^{\prime}} \mid v_{2, j} \in L_{2, k}\right\rangle$.

Proof Using [14, Proposition VI.2.1] on $S_{2}$, we get that $\varphi_{2}=\prod_{j=10}^{1} C_{2, j} \widetilde{\varphi}_{2, j} \prod b_{\ell}$. $h_{j} \in\left\langle Z_{k k^{\prime}} \mid v_{2, j} \in L_{2, k}\right\rangle$ are determined by the regeneration of the embedding $B_{k} \hookrightarrow$ $B_{24}$ to $B_{2 k} \hookrightarrow B_{48}$ where $k=4$ when $j=5,6$ and $k=5$ otherwise $(1 \leq j \leq 10, j \neq$ 5,6 ; see the definition of regeneration of an embedding in [16, Section 1]). $b_{\ell}$ are factors that are not converted by $\prod C_{2, j} \widetilde{\varphi}_{2, j}$, and each $b_{n}$ is of the form $Y_{i}^{t_{i}}, Y_{i}$, is a positive half-twist, $0 \leq t_{i} \leq 3$. Note that $\operatorname{deg}\left(\widetilde{\varphi}_{2, j}\right)=\operatorname{deg}\left(\varphi_{2, j}\right)$. By the previous proposition, we know part of the $b_{\ell}$ 's; so we can say that $\varphi_{2}=\prod_{j=10}^{1} C_{2, j} \widetilde{\varphi}_{2, j} \prod_{n=1}^{16} b_{n} \prod b_{\ell}$. We compute $\operatorname{deg}\left(\prod_{j=10}^{1} C_{2, j} \widetilde{\varphi}_{2, j} \prod_{n=1}^{16} b_{n}\right)$. By earlier computations and the previous proposition,

$\operatorname{deg}\left(\prod_{j=10}^{1} C_{2, j} \widetilde{\varphi}_{2, j} \prod_{n=1}^{16} b_{n}\right)=2232+2 \cdot 8+8=2256=48 \cdot 47=\operatorname{deg}\left(\Delta_{48}^{2}\right)=\operatorname{deg} \varphi_{2}$.

Thus, we have to compute $\operatorname{deg}\left(\prod b_{\ell}\right)$. Since $\forall \ell, b_{\ell}$ is a positive power of a positive half-twist, we get $b_{\ell}=1 \forall \ell$. So we have

$$
\varphi_{2}=\prod_{j=10}^{1} C_{2, j} \widetilde{\varphi}_{2, j} \prod_{n=1}^{16} b_{n}
$$

Algebraic 83 Geometric Topology, Volume 8 (2008) 
Lemma 3.10 $\varphi_{2}=\prod_{j=10}^{1} C_{2, j} \varphi_{2, j} \prod_{n=1}^{16} b_{n}$.

Proof Recalling the invariance rules for the BMF of 4- and 5- point (see [16] and

Section 5), we can apply them as in [16], and get that $\prod_{j=10}^{1} C_{2, j} \varphi_{2, j} \prod_{n=1}^{16} b_{n}$ is also a braid monodromy factorization.

Note that although the invariance rules for the 5-point are different from the invariance rules of the standard $4 / 6$ - point, what matters, as can be seen in [16, Section 4] is that the invariance rule regarding the horizontal lines in the 5-point (the two lines that are regenerated last) remains the same in this type of point.

\section{Computing the fundamental groups}

\subsection{Computation for $X_{2}$}

By the Van Kampen theorem (Theorem 2.5), we can compute the relations between the generators in the fundamental group of the complement of the branch curve.

We will prove that $\pi_{1}\left(\mathbb{C}-S_{2}\right)$ is a quotient of $\widetilde{B}_{16}$. In order to do so, we have to compute the local relations (or the local fundamental groups of the complement of the branch curve) arising from each singular point of the branch curve. Note that points $v_{2,5}, v_{2,6}$ are of the type 4-point, which was investigated by Moishezon [11] and Robb [18]. Thus, we have to look at the remaining 5-points. We focus only on one 5-point $v_{2,3}$; for the other 5-points, the procedure for deducing the relations is the same, and we state (later) only the relations coming from the branch points for these points.

Recall that in the regeneration process, every line is "doubled", and thus $S_{i} \cap \mathbb{C}$ will contain $48=2 \cdot 24$ points. The generators of $\pi_{1}\left(\mathbb{C}^{2}-S, u\right)$ (see the Van Kampen Theorem, Theorem 2.5) induced from this doubling are denoted as $\left\{\Gamma_{i}, \Gamma_{i^{\prime}}\right\}_{i=1}^{24}$, where each pair $\left\{\Gamma_{i}, \Gamma_{i^{\prime}}\right\}$ originates from the same line.

Denote $\bar{\Gamma}_{i}=\Gamma_{i}$ or $\Gamma_{i^{\prime}}$. Before examining $\varphi_{2,3}$ we state the following remark.

Remark 4.1 $\forall_{i, j}$ s.t. $L_{2, i} \bigcap L_{2, j}=\varnothing$, we have the following relations in $\pi_{1}\left(\mathbb{C}^{2}-\right.$ $\left.S_{2}\right)$ :

$$
\left[\bar{\Gamma}_{i}, \bar{\Gamma}_{j}\right]=1
$$

The proof of this remark is based on the parasitic intersection braids. From each braid of the expressions $C_{2, i}(i=1, \ldots, 10)$, using complex conjugation and the Van Kampen Theorem, we can induce the above relations. 
Proposition 4.2 The following relations in $\pi_{1}\left(\mathbb{C}^{2}-S_{2}\right)$ are induced from $\varphi_{2,3}$ :

(i) $\left\langle\bar{\Gamma}_{16}, \bar{\Gamma}_{1}\right\rangle=\left\langle\bar{\Gamma}_{1}, \bar{\Gamma}_{9}\right\rangle=\left\langle\bar{\Gamma}_{9}, \bar{\Gamma}_{21}\right\rangle=\left\langle\bar{\Gamma}_{21}, \bar{\Gamma}_{3}\right\rangle=\left\langle\bar{\Gamma}_{3}, \bar{\Gamma}_{16}\right\rangle=1$

(ii) $\left[\bar{\Gamma}_{i}, \bar{\Gamma}_{j}\right]=1$ where $L_{i}, L_{j}$ do not bound the same triangle, besides $\left(i=1^{\prime}, j=\right.$ $3),\left(i=1, j=3^{\prime}\right)$ and $(i=1, j=21)$

(iii) $\Gamma_{1}=\left(\Gamma_{3^{\prime}}\right)_{\Gamma_{16}^{-1}}^{-1} \Gamma_{21}^{-1} \Gamma_{9}$.

Proof In the course of the proof we use the Van Kampen Theorem, the invariance relations of the 5-point, and the complex conjugation method (see [16]). We prove the proposition in several steps.

Step 1 By looking at the braids (in $\varphi_{2,3}$ ) $Z_{33^{\prime}, 9}^{2}, Z_{99^{\prime}, 16}^{2}, Z_{11^{\prime}, 9}^{(3)}$ and $\bar{Z}_{9^{\prime}, 2121^{\prime}}^{(3)}$, we induce immediately (using invariance relations and complex conjugation for the last braid) the following relations:

$$
\left[\bar{\Gamma}_{9}, \bar{\Gamma}_{16}\right]=\left[\bar{\Gamma}_{3}, \bar{\Gamma}_{9}\right]=\left\langle\bar{\Gamma}_{1}, \bar{\Gamma}_{9}\right\rangle=\left\langle\bar{\Gamma}_{9}, \bar{\Gamma}_{21}\right\rangle=1 .
$$

Step 2 Note that the factors in $\left(F_{3} \cdot\left(F_{3}\right)_{\vartheta}\right)$ are conjugated by $Z_{\alpha_{3}}$. Denote the corresponding generators induced from $\left(F_{3} \cdot\left(F_{3}\right)_{\vartheta}\right)$ (after the conjugation) by $\widetilde{\Gamma}_{i}$.

So:

$$
\begin{array}{ll}
\widetilde{\Gamma}_{3}=\Gamma_{9} \Gamma_{3} \Gamma_{9}^{-1} \stackrel{\text { by step } 1}{=} \Gamma_{3} & \widetilde{\Gamma}_{3^{\prime}}=\Gamma_{9} \Gamma_{3^{\prime}} \Gamma_{9}^{-1}=\Gamma_{3^{\prime}} \\
\widetilde{\Gamma}_{1}=\Gamma_{9} \Gamma_{1} \Gamma_{9}^{-1} & \widetilde{\Gamma}_{1^{\prime}}=\Gamma_{9} \Gamma_{1^{\prime}} \Gamma_{9}^{-1},
\end{array}
$$

the other $\widetilde{\Gamma}_{i}$ are not changed. So, we have, by the braid $Z_{33^{\prime}, 16}^{(3)}$ in $F_{3}$ the relation

$$
\left\langle\bar{\Gamma}_{3}, \bar{\Gamma}_{16}\right\rangle=1 .
$$

Step 3 From the braid $Z_{16^{\prime}, 21}^{2}$ in $F_{3}$, we get the relation: $\left[\Gamma_{16^{\prime}}, \Gamma_{21}\right]=1$. Looking on the complex conjugate of the braid $\left(Z_{16^{\prime}, 21}^{2}\right)_{\vartheta}$, we now get the relation

$$
\left[\Gamma_{16}, \Gamma_{21}^{-1} \Gamma_{21^{\prime}} \Gamma_{21}\right]=1 \stackrel{\text { (inv. rel. } \left.\rho_{16} \rho_{21}\right)}{\longrightarrow}\left[\Gamma_{16^{\prime}}, \Gamma_{21}^{-2} \Gamma_{21^{\prime}} \Gamma_{21}^{2}\right]=\left[\Gamma_{16^{\prime}}, \Gamma_{21^{\prime}}\right]=1 .
$$

By performing another time the invariance relation $\left(\rho_{16} \rho_{21}\right)$, we get $\left[\Gamma_{16}, \Gamma_{21}\right]=1$. From $\left[\Gamma_{16^{\prime}}, \Gamma_{21}^{-1} \Gamma_{21^{\prime}} \Gamma_{21}\right]=1$, we get $\left[\Gamma_{16}, \Gamma_{21^{\prime}}\right]=1$.

So we have the relation $\left[\bar{\Gamma}_{16}, \bar{\Gamma}_{21}\right]=1$.

Step 4 From the braid $\left(Z_{33^{\prime}, 21}^{(3)}\right)_{Z_{33^{\prime}, 16}^{(3)}}$, we get the relation: $\left\langle\Gamma_{21}, \Gamma_{16} \Gamma_{3^{\prime}} \Gamma_{16}^{-1}\right\rangle=1$. By step 3 we get $\left\langle\Gamma_{21}, \Gamma_{3^{\prime}}\right\rangle=1$; in the same way, we get $\left\langle\Gamma_{21}, \Gamma_{3^{\prime}}\right\rangle=1$ and by invariance relation, we get: $\left\langle\bar{\Gamma}_{21}, \bar{\Gamma}_{3}\right\rangle=1$. 
Step 5 From the braid $\left(\left(Z_{1,3^{\prime}}\right)_{Z_{3^{\prime}, 21}^{2}} Z_{3^{\prime}, 16}^{2}\right)_{\alpha_{3}}$, we get the relation:

$$
\Gamma_{1}=\left(\Gamma_{3^{\prime}}\right)_{16}^{-1} \Gamma_{21}^{-1} \Gamma_{9}
$$

Thus

$$
\begin{gathered}
\left\langle\Gamma_{16}, \Gamma_{1}\right\rangle=\left\langle\Gamma_{16},\left(\Gamma_{3^{\prime}}\right)_{\Gamma_{16}^{-1} \Gamma_{21}^{-1} \Gamma_{9}}\right\rangle=\left\langle\left(\Gamma_{16}\right)_{\Gamma_{21}},\left(\Gamma_{3^{\prime}}\right)_{\Gamma_{16}^{-1}}^{-1}\right\rangle \\
{\left[\Gamma_{16}, \stackrel{\left.\Gamma_{21}\right]=1}{=}\left\langle\Gamma_{16}, \Gamma_{16} \Gamma_{3^{\prime}} \Gamma_{16}^{-1}\right\rangle=\left\langle\Gamma_{16}, \Gamma_{3^{\prime}}\right\rangle=1 .\right.}
\end{gathered}
$$

By the invariance relations, we get: $\left\langle\bar{\Gamma}_{16}, \bar{\Gamma}_{1}\right\rangle=1$.

Step 6 We know that $\Gamma_{1}=\left(\Gamma_{3^{\prime}}\right)_{\Gamma_{16}^{-1}} \Gamma_{21}^{-1} \Gamma_{9}$ and thus $\left(\Gamma_{1}\right)_{\Gamma_{9}^{-1} \Gamma_{16}}=\left(\Gamma_{3^{\prime}}\right)_{\Gamma_{21}^{-1}}$ (by $\left.\left[\Gamma_{16}, \Gamma_{21}\right]=1\right)$. From the braid $\left(\left(Z_{16,21}^{2}\right)_{Z_{33^{\prime}, 16}^{2}}\right)_{Z_{\alpha_{3}}}$, we get the relation:

$$
\begin{aligned}
& {\left[\Gamma_{16},\left(\Gamma_{21}\right)_{\left.\Gamma_{3^{\prime}} \Gamma_{3}\right]}\right] }=1 \text { or } \\
& 1=\left[\Gamma_{3} \Gamma_{16} \Gamma_{3}^{-1}, \Gamma_{3^{\prime}}^{-1} \Gamma_{21} \Gamma_{3^{\prime}}\right] \\
&\left\langle\Gamma_{3}, \Gamma_{16}\right\rangle=\left\langle\Gamma_{3^{\prime}}, \Gamma_{21}\right\rangle 1 {\left[\Gamma_{16}^{-1} \Gamma_{3} \Gamma_{16}, \Gamma_{21} \Gamma_{3^{\prime}} \Gamma_{21}^{-1}\right] } \\
&= {\left[\Gamma_{16}^{-1} \Gamma_{3} \Gamma_{16}, \Gamma_{16}^{-1} \Gamma_{9} \Gamma_{1} \Gamma_{9}^{-1} \Gamma_{16}^{-1}\right] } \\
& {\left[\Gamma_{9}, \Gamma_{16}\right] \stackrel{\left[\Gamma_{9}, \Gamma_{3}\right]}{=}=1\left[\Gamma_{3}, \Gamma_{1}\right] }
\end{aligned}
$$

and by invariance we get $\left[\Gamma_{3^{\prime}}, \Gamma_{1^{\prime}}\right]=1$.

The following proposition proves the missing relations $\left(\mathrm{eg},\left[\bar{\Gamma}_{1}, \bar{\Gamma}_{21}\right]=1\right)$. The reason for separating this proposition from the former is because we use now relations which are not necessarily from $\varphi_{2,3}$.

Proposition 4.3 The following relations in $\pi_{1}\left(\mathbb{C}^{2}-S_{2}\right)$ hold

$$
\left[\bar{\Gamma}_{1}, \bar{\Gamma}_{3}\right]=\left[\bar{\Gamma}_{1}, \bar{\Gamma}_{21}\right]=1 \text {. }
$$

Proof Due to the invariance relations of $v_{2,3}$, it is enough to prove $\left[\Gamma_{1}, \Gamma_{3^{\prime}}\right]=1$ and $\left[\Gamma_{1}, \Gamma_{21}\right]=1$.

By the braid $Z_{3,3^{\prime}}$ (induced from an extra branch point), we know that $\Gamma_{3}=\Gamma_{3^{\prime}}$. Thus, by the last Proposition 4.2, step 6, we have

$$
1=\left[\Gamma_{1}, \Gamma_{3}\right]=\left[\Gamma_{1}, \Gamma_{3^{\prime}}\right]
$$

Looking on the local BMF of $v_{2,1}$, we have the following relation from the braid $\left(\left(Z_{1,5^{\prime}}\right)_{5^{\prime}, 11}^{2} Z_{5^{\prime}, 7}^{2}\right)_{1111^{\prime}, 15}^{2}$ :

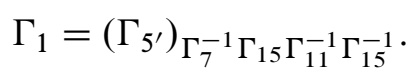


Since $\Gamma_{21}$ commutes with $\Gamma_{5^{\prime}}, \Gamma_{7}, \Gamma_{15}$ and $\Gamma_{11}$ (due to the parasitic intersection braids), we have that $\left[\Gamma_{1}, \Gamma_{21}\right]=1$.

Proposition 4.4 $\forall i, 1 \leq i \leq 24, i \neq 9,13,15,20, \Gamma_{i}=\Gamma_{i^{\prime}}$ in $\pi_{1}\left(\mathbb{C}^{2}-S_{2}\right)$.

We divide the proof into two lemmas.

Lemma 4.5 For $i=3, \ldots, 8,10,11,12,14,16,17,18,21,22,23: \Gamma_{i}=\Gamma_{i^{\prime}}$.

Proof The relation $\Gamma_{i}=\Gamma_{i^{\prime}}$ is induced from the braids $Z_{i, i^{\prime}}$ which are created from the extra branch points (by Proposition 3.8).

Lemma 4.6 For $i=1,2,19,24: \Gamma_{i}=\Gamma_{i^{\prime}}$.

Proof We will prove in details only for $i=1$; the proof for the other $i$ is the same. We know (from the braid $\left(\left(Z_{1,5^{\prime}}\right)_{5^{\prime}, 7}^{2} Z_{5^{\prime}, 11}^{2}\right)_{Z_{1111^{\prime}, 15}^{2}}$ in $\left.\varphi_{2,1}\right)$ the relation: $\Gamma_{1}=$ $\Gamma_{15} \Gamma_{11} \Gamma_{7} \Gamma_{5} \Gamma_{7}^{-1} \Gamma_{11}^{-1} \Gamma_{15}^{-1}$ (we used the relation $\Gamma_{5}=\Gamma_{5}$ ). Operating the invariance relations $\left(\rho_{1} \rho_{5}\right)\left(\rho_{7} \rho_{11}\right)$ and taking the inverse, we get:

$$
\Gamma_{1}^{-1}=\Gamma_{15} \Gamma_{11^{\prime}} \Gamma_{7^{\prime}} \Gamma_{5^{\prime}}^{-1} \Gamma_{7^{\prime}}^{-1} \Gamma_{11^{\prime}}^{-1} \Gamma_{15}^{-1} .
$$

Multiplying the above relations and using Lemma 4.5, we get $\Gamma_{1^{\prime}}^{-1} \Gamma_{1}=1$, or $\Gamma_{1}=\Gamma_{1^{\prime}}$.

For $i=2$, we use the braid $\left(\left(Z_{2,6^{\prime}}\right)_{Z_{6^{\prime}, 8}^{2} Z_{6^{\prime}, 12}^{2}}\right) Z_{\alpha_{2}}$ from $\varphi_{2,2}$ and the same method as above.

For $i=19,24$, one can use the braids $\left(\left(\bar{Z}_{10^{\prime}, 24}\right)_{\ldots}\right)_{Z_{\alpha_{7}}}$ from $\varphi_{2,7}$ (or the braid $\left(\left(\bar{Z}_{14^{\prime}, 19}\right)_{\ldots}\right)_{\alpha_{\alpha_{8}}}$ from $\left.\varphi_{2,8}\right)$ and continue as above.

Remark 4.7 For each $1 \leq i \leq 10$ we denote by $G_{2, i}$ the local fundamental whose generators are $\Gamma_{j}$, such that one of the endpoints of $L_{2, j}$ is $v_{2, i}$. Generalizing Proposition 4.2 and Proposition 4.3, it is easy to prove that $\forall_{i, j}$ s.t. $L_{2, i}$ and $L_{2, j}$ do not bound a common triangle, $\left[\bar{\Gamma}_{i}, \bar{\Gamma}_{j}\right]=1$; and $\forall_{i, j}$ s.t. $L_{2, i}$ and $L_{2, j}$ bound a common triangle, $\left\langle\bar{\Gamma}_{i}, \bar{\Gamma}_{j}\right\rangle=1\left(\right.$ in $\pi_{1}\left(\mathbb{C}^{2}-S_{2}\right)$ ).

Remark 4.8 It is important to state which braids are coming from the branch points. We list below (for each $\varphi_{2, i}$, for $1 \leq i \leq 10, i \neq 5,6$ ) which braid is induced from 
a branch point, that is created during the regeneration of the horizontal lines of the 5-point. We use the double and triple relations, and the last proposition, and we obtain

$$
\begin{array}{ll}
i=1: & \Gamma_{1}=\left(\Gamma_{5}\right)_{\Gamma_{7}^{-1} \Gamma_{11}^{-1} \Gamma_{15}^{-1}} \\
i=2: & \Gamma_{2}=\left(\Gamma_{6}\right) \Gamma_{8}^{-1} \Gamma_{12}^{-1} \Gamma_{20}^{-1} \\
i=3: & \Gamma_{1}=\left(\Gamma_{3}\right)_{16}^{-1} \Gamma_{21}^{-1} \Gamma_{9} \\
i=4: & \Gamma_{2}=\left(\Gamma_{4}\right)_{\Gamma_{17}^{-1} \Gamma_{13} \Gamma_{22}^{-1}} \\
i=7: & \Gamma_{24}=\left(\Gamma_{10}\right)_{\Gamma_{7}^{-1} \Gamma_{8}^{-1} \Gamma_{9}^{-1}} \\
i=8: & \Gamma_{19}=\left(\Gamma_{14}\right)_{11} \Gamma_{12} \Gamma_{13} \\
i=9: & \Gamma_{18}=\left(\Gamma_{17}\right)_{\Gamma_{19}} \Gamma_{15^{\prime}} \Gamma_{16}^{-1} \\
i=10: & \Gamma_{23}=\left(\Gamma_{24}\right)_{\Gamma_{20^{\prime}} \Gamma_{22}^{-1} \Gamma_{21}} .
\end{array}
$$

Proposition 4.9 For $i=5,6$, there exist a homomorphism $\alpha_{i}: \widetilde{B}_{4} \rightarrow G_{2, i}$.

Proof This proposition is proven in [18].

Proposition 4.10 For $1 \leq i \leq 10, i \neq 5,6$, there exist a homomorphism $\alpha_{i}$ : $\widetilde{B}_{5} \rightarrow$ $G_{2, i}$.

Proof Using the Remark 4.8 we prove only for $i=1$, and the proof for the other $i$ is done in the same way.

It is easy to check that $\alpha_{1}: B_{5} \rightarrow G_{2,1}$ is well-defined:

$$
\alpha_{1}\left(X_{1}\right)=\Gamma_{7} \quad \alpha_{1}\left(X_{2}\right)=\Gamma_{5} \quad \alpha_{1}\left(X_{3}\right)=\Gamma_{11} \quad \alpha_{1}\left(X_{4}\right)=\Gamma_{15} .
$$

Let $x_{1}, \ldots, x_{4}$ be the images of $X_{1}, \ldots, X_{4}$ in $\widetilde{B}_{5}$. Consider

$$
T=X_{4} X_{3} X_{1} X_{2} X_{1}^{-1} X_{3}^{-1} X_{4}^{-1}
$$

in $B_{5}$ (see Figure 21).

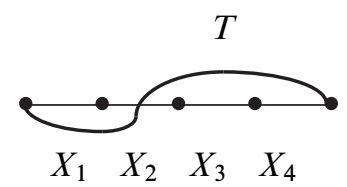

Figure 21

Algebraic $8 \mathcal{G}$ Geometric Topology, Volume 8 (2008) 
$T$ is transversal to $X_{2}$. Let $t$ be the image of $T$ in $\widetilde{B}_{5}$; by the definition of $\widetilde{B}_{5}$ we have $\left[t, x_{2}\right]=1$. To show that $\alpha_{1}: B_{5} \rightarrow G_{2,1}$ defines the desired $\alpha_{1}: \widetilde{B}_{5} \rightarrow G_{2,1}$, it is enough to check that

$$
\left[\alpha_{1}(T), \alpha\left(X_{2}\right)\right]=1 .
$$

We claim that $\alpha(T)=\Gamma_{1}$, because

$$
\alpha(T)=\alpha\left(X_{4} X_{3} X_{1} X_{2} X_{1}^{-1} X_{3}^{-1} X_{4}^{-1}\right)=\left(\Gamma_{5}\right)_{\Gamma_{7}^{-1} \Gamma_{11}^{-1} \Gamma_{15}^{-1}}=\Gamma_{1} .
$$

So we have $\left[\alpha(T), \alpha\left(X_{2}\right)\right]=\left[\Gamma_{1}, \Gamma_{5}\right]=1$.

The last proposition deals with the relations between $\Gamma_{i^{\prime}}$ and $\Gamma_{i}$ in $\pi_{1}\left(\mathbb{C}^{2}-S_{2}\right)$, where $i=9,13,15,20$.

Proposition 4.11 The following relations in $\pi_{1}\left(\mathbb{C}^{2}-S_{2}\right)$ hold:

(i) $\Gamma_{13^{\prime}}=\left(\Gamma_{13}^{2}\right)_{\Gamma_{17}^{2}} \Gamma_{13}^{-1}$,

(ii) $\Gamma_{9^{\prime}}=\left(\Gamma_{9}^{2}\right)_{\Gamma_{21}^{2}} \Gamma_{9}^{-1}$,

(iii) $\Gamma_{15^{\prime}}=\Gamma_{15}$,

(iv) $\Gamma_{20^{\prime}}=\Gamma_{20}$

\section{Proof}

(i) From the braid $\widetilde{Z}_{13,13^{\prime}}$ in $\varphi_{2,3}$, we induce:

$$
\Gamma_{13}=\Gamma_{2}^{-1} \Gamma_{2^{\prime}}^{-1} \Gamma_{4}^{-1} \Gamma_{4^{\prime}}^{-1} \Gamma_{13}^{-1} \Gamma_{17^{\prime}}^{-1} \Gamma_{17} \Gamma_{13^{\prime}} \Gamma_{17}^{-1} \Gamma_{17^{\prime}}^{-1} \Gamma_{13} \Gamma_{4^{\prime}} \Gamma_{4} \Gamma_{2^{\prime}} \Gamma_{2} .
$$

Using $\left[\bar{\Gamma}_{4}, \bar{\Gamma}_{2}\right]=\left[\bar{\Gamma}_{4}, \bar{\Gamma}_{13}\right]=1$ and $\Gamma_{2}=\Gamma_{2^{\prime}}, \Gamma_{17}=\Gamma_{17^{\prime}}$, we get

$$
\begin{aligned}
& 1=\Gamma_{2}^{-2} \Gamma_{13}^{-1} \Gamma_{17}^{2} \Gamma_{13^{\prime}} \Gamma_{17}^{-2} \Gamma_{13} \Gamma_{2}^{2} \Gamma_{13}^{-1} \text { or : } \\
& 1=\Gamma_{2}^{-2}\left(\Gamma_{17}^{2}\right)_{13} \cdot\left(\Gamma_{13}^{-1} \Gamma_{13^{\prime}}\right) \Gamma_{17}^{-2}\left(\Gamma_{2}^{2}\right)_{\Gamma_{13}^{-1}} .
\end{aligned}
$$

By $\left[\bar{\Gamma}_{2}, \bar{\Gamma}_{17}\right]=1$, we get

$$
\begin{aligned}
\Gamma_{13}^{-1} \Gamma_{13^{\prime}} & =\left(\Gamma_{17}^{-2}\right)_{\Gamma_{13}}\left(\Gamma_{2}^{-2}\right)_{\Gamma_{13}^{-1}} \Gamma_{2}^{2} \Gamma_{17}^{2} \\
& =\left(\Gamma_{17}^{-2}\right)_{\Gamma_{13}}(\left(\Gamma_{17}^{-2}\right)_{\Gamma_{13}^{-1}}^{-1} \underbrace{\left(\Gamma_{17}^{-2}\right)_{\Gamma_{13}^{-1}}\left(\Gamma_{2}^{-2}\right)_{\Gamma_{13}^{-1}} \Gamma_{2}^{2} \Gamma_{17}^{2}}_{F_{13}} .
\end{aligned}
$$


By Proposition 4.10, the braids in $F_{13}: \Gamma_{2}, \Gamma_{17},\left(\Gamma_{2}\right)_{\Gamma_{13}^{-1}},\left(\Gamma_{17}\right)_{\Gamma_{13}^{-1}}$ are images of a good quadrangle by $\alpha_{4}$ in $\widetilde{B}_{5}$, and thus $F_{13}=1$ (by Lemma 2.6 on good quadrangles in $\widetilde{B}_{n}$ ). Thus

$$
\begin{aligned}
\Gamma_{13}^{-1} \Gamma_{13^{\prime}} & =\Gamma_{13}^{-1} \Gamma_{17}^{-2} \Gamma_{13}^{2} \Gamma_{17}^{2} \Gamma_{13}^{-1} \\
& \text { or } \Gamma_{13^{\prime}}=\left(\Gamma_{13}^{-2}\right)_{\Gamma_{17}^{-2}}^{-2} \cdot \Gamma_{13}^{-1} .
\end{aligned}
$$

(ii) We apply the same procedure as in (i) to the braid $\widetilde{Z}_{9,9^{\prime}}$ from $\varphi_{2,3}$.

(iii) Taking the complex conjugate of $\widetilde{Z}_{15,15^{\prime}}$ in $\varphi_{2,1}$, we induce the relation (using $\left.\Gamma_{1}=\Gamma_{1^{\prime}}, \Gamma_{11}=\Gamma_{11^{\prime}}\right)$ :

$$
\Gamma_{15^{\prime}}=\Gamma_{1}^{-2} \Gamma_{11}^{-2} \Gamma_{15} \Gamma_{11}^{2} \Gamma_{1}^{2}
$$

or

$$
\begin{aligned}
1 & =\Gamma_{11}^{2} \Gamma_{1}^{2}\left(\Gamma_{15^{\prime}} \Gamma_{15}^{-1}\right)\left(\Gamma_{1}^{-2}\right)_{\Gamma_{15}^{-1}}\left(\Gamma_{11}^{-2}\right)_{\Gamma_{15}^{-1}} \\
\Gamma_{15} \cdot \Gamma_{15^{\prime}}^{-1} & =\left(\Gamma_{1}^{-2}\right)_{\Gamma_{15}^{-1}}\left(\Gamma_{11}^{2}\right)_{\Gamma_{15}^{-1}} \Gamma_{11}^{2} \Gamma_{1}^{2} .
\end{aligned}
$$

By the same method as in (i) (using $\alpha_{1}: \widetilde{B}_{5} \rightarrow G_{2,1}$ ), we get that

$$
\Gamma_{15} \Gamma_{15^{\prime}}^{-1}=1 \text { or } \Gamma_{15}=\Gamma_{15^{\prime}} .
$$

(iv) Taking the complex conjugation of $\widetilde{Z}_{20,20^{\prime}}$ in $\varphi_{2,2}$, we induce the relation (using $\Gamma_{2}=\Gamma_{2^{\prime}}, \Gamma_{8}=\Gamma_{8^{\prime}}$ ), and $\left[\bar{\Gamma}_{12}, \bar{\Gamma}_{20}\right]=1$ ):

$$
\Gamma_{20^{\prime}}=\Gamma_{2}^{-2} \Gamma_{8}^{-2} \Gamma_{20} \Gamma_{8}^{2} \Gamma_{2}^{2}
$$

and we proceed as in (iii). Thus: $\Gamma_{20}=\Gamma_{20^{\prime}}$.

These propositions show that $\pi_{1}\left(\mathbb{C}^{2}-S_{2}\right)$ is generated only by $\left\{\Gamma_{i}\right\}_{i=1}^{24}$, since the $\left\{\Gamma_{i^{\prime}}\right\}_{i=1}^{24}$ can be expressed only in terms of the $\left(\Gamma_{i^{\prime}}\right)$. Our last goal is to prove the following theorem.

Theorem 4.12 $G_{2}=\pi_{1}\left(\mathbb{C}-S_{2}\right)$ is a quotient of $\widetilde{B}_{16}$.

Proof We need to build an epimorphism $\widetilde{\alpha}: \widetilde{B}_{16} \rightarrow G_{2}$. But first we build a new representation for $B_{16}$. Consider the geometric model $(D, K), \# K=16$ as in Figure 22. Let $\left\{t_{i}\right\}_{i \in I}, I=\{1 \leq i \leq 24, i \neq 1,3,5,8,11,12,16,17,22, i \in \mathbb{Z}\}$ segments that connect points in $K$ and $T_{i}$ be the half-twists corresponding to $t_{i}$ (that is, $T_{i}=$ $\left.H\left(t_{i}\right), i \in I\right)$. 


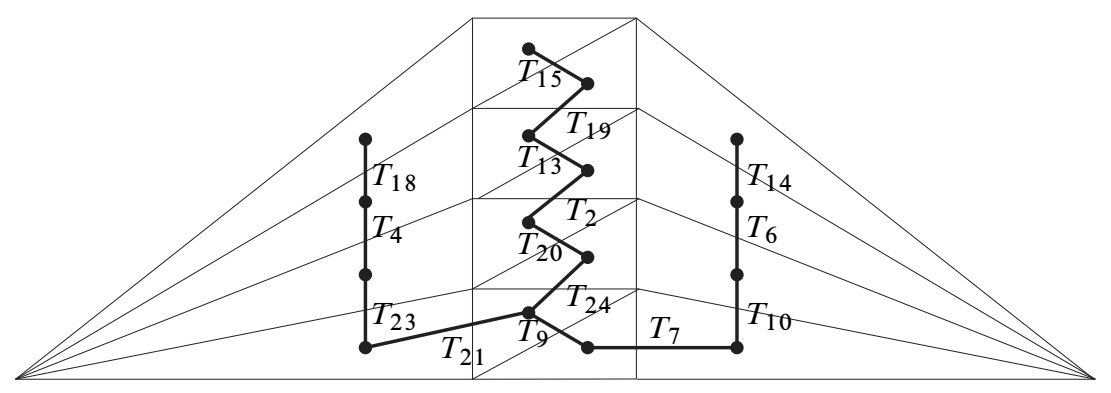

Figure 22

Lemma 4.13 There exists a presentation of $B_{16}$ when the generators are $\left\{T_{i} \mid i \in I\right\}$ and the relations are:

$$
\begin{aligned}
\left\langle T_{i}, T_{j}\right\rangle & =1 \text { if } T_{i}, T_{j} \text { are consecutive, } \\
{\left[T_{i}, T_{j}\right] } & =1 \text { if } T_{i}, T_{j} \text { are disjoint, } \\
{\left[T_{9}, T_{24} T_{21} T_{24}^{-1}\right] } & =1 .
\end{aligned}
$$

Proof This is a standard consequence of the usual presentation of $B_{16}(D, K)$ (see [14]).

Let $X, Y \in B_{16}$ be transversal half-twists and let $\widetilde{B}_{16}=B_{16} /\langle[X, Y]\rangle$. By the previous lemma, $\widetilde{B}_{16}$ is generated by $\left\{\widetilde{T}_{i}\right\}_{i \in I}$ (where $\widetilde{T}_{i}$ are the images of $T_{i}$ in $\widetilde{B}_{16}$ ), and has same relations.

Using Remark 4.8 we can define the "missing" $T_{j}$ (where $1 \leq j \leq 24, j \notin I$ ). We begin with $j=8,11,12,16,17,22$ :

$$
T_{8}=\left(T_{10}\right)_{T_{7}^{-1} T_{9}^{-1} T_{24}} T_{12}=\left(T_{6}\right)_{T_{8}^{-1} T_{20}^{-1} T_{2}} T_{11}=\left(T_{14}\right)_{T_{12} T_{13} T_{19}^{-1}}
$$

(we can use $T_{8}$ and $T_{12}$ since these $T$ 's are already defined)

$$
T_{22}=\left(T_{24}\right)_{T_{20} T_{21} T_{23}} \quad T_{17}=\left(T_{2}\right)_{T_{22} T_{3}^{-1} T_{4}} T_{16}=\left(T_{17}\right)_{T_{19}^{-1} T_{15} T_{18}}
$$

(we used $\left.\Gamma_{20}=\Gamma_{20^{\prime}}\right)$.

In order to find out how to define $T_{3}$ (and $T_{5}$ ), we look at a relation induced from $\varphi_{2,5}\left(\varphi_{2,6}\right)$. Looking at the braid $\left(Z_{3,4^{\prime}}\right)_{4_{4^{\prime}, 23}} Z_{4^{\prime}, 18}^{2}$ from $\varphi_{2,5}$, we get the relation $\Gamma_{3}=\left(\Gamma_{4}\right)_{\Gamma_{18}^{-1}} \Gamma_{23}^{-1}$. Thus we define $T_{3}=\left(T_{4}\right)_{T_{18}^{-1} T_{23}^{-1}}$. In the same way we define $T_{5}=\left(T_{6}\right)_{T_{10}^{-1}} T_{14}^{-1}$. By Remark 4.8, let $T_{1}=\left(T_{5}\right)_{T_{7}^{-1}} T_{11}^{-1} T_{15}^{-1}$. 
Denoting by $\left\{\widetilde{T}_{j}\right\}_{j=1}^{24}$ the images of $\left\{T_{j}\right\}_{j=1}^{24}$ in $\widetilde{B}_{16}$, we can say that $\widetilde{B}_{16}$ is generated by $\left\{\tilde{T}_{j}\right\}_{j=1}^{24}$ with the same relations as above, and when the $\left\{\tilde{T}_{j}\right\}_{j \notin I}$ are defined as above. Define $\widetilde{\alpha}\left(\widetilde{T}_{j}\right)=\Gamma_{j}, 1 \leq j \leq 24$. By Remark 4.7, it is easy to see that $\forall i, j$ such that $T_{i}$ and $T_{j}$ are consecutive, $\left\langle\Gamma_{i}, \Gamma_{j}\right\rangle=1$; and when $T_{i}$ and $T_{j}$ are disjoint, $\left[T_{i}, T_{j}\right]=1$. The relations induced from the action of taking quotient by $\langle[X, Y]\rangle$ (when $X, Y \in B_{16}$ are transversal) are also preserved, due to Proposition 4.9 and Proposition 4.10. Also, $\widetilde{\alpha}$ is an epimorphism, since for every generator $\Gamma_{j}$ of $G_{2}$ there exists a $\widetilde{T}_{j}$ s.t. $\widetilde{\alpha}\left(\widetilde{T}_{j}\right)=\Gamma_{j}$. Thus $G_{2} \simeq \widetilde{B}_{16} / \operatorname{ker} \widetilde{\alpha}$.

\subsection{Computation for $X_{1}$}

As in Section 4.1, we can compute the local relations induced from each local braid monodromy. However, a quotient of the fundamental group of $X_{1}-$ called the stabilized fundamental group - was already computed in Auroux-Donaldson-Katzarkov-Yotov [4]. Noticing that $X_{1}$ can be regarded as a double cover of $\mathbb{C P} \mathbb{P}^{1} \times \mathbb{C P}^{1}$ branched along a smooth algebraic curve of degree $(4,4)$, we can use [4, Theorem 4.6].

Let $\Theta_{1}: \pi_{1}\left(\mathbb{C}^{2}-S_{1}\right) \rightarrow S_{n}$ be the geometric monodromy representation morphism (here $n=16$ ).

Theorem 4.14 Let $K_{1}$ be the normal subgroup of $\pi_{1}\left(\mathbb{C}^{2}-S_{1}\right)$ generated by all commutators $\left[\gamma_{1}, \gamma_{2}\right], \gamma_{1}, \gamma_{2}$ - geometric generators of $\pi_{1}\left(\mathbb{C}^{2}-S_{1}\right)$, such that $\Theta_{1}\left(\gamma_{1}\right)$ and $\Theta_{1}\left(\gamma_{2}\right)$ are disjoint transpositions. Let

$$
G_{1}^{0} \doteq\left(\operatorname{ker}\left(\Theta_{1}: \pi_{1}\left(\mathbb{C}^{2}-S_{1}\right) \rightarrow S_{n}\right) \bigcap \operatorname{ker}\left(\operatorname{deg}: \pi_{1}\left(\mathbb{C}^{2}-S_{1}\right) \rightarrow \mathbb{Z}\right)\right) / K_{1},
$$

where deg is the degree morphism. Then

$$
\operatorname{Ab}\left(G_{1}^{0}\right) \simeq\left(\mathbb{Z} \oplus \mathbb{Z}_{2}\right)^{15} \text { and }\left[G_{1}^{0}, G_{1}^{0}\right] \simeq \mathbb{Z}_{2} \oplus \mathbb{Z}_{2} .
$$

Proof See [4, Theorem 4.6].

The group $\pi_{1}\left(\mathbb{C}^{2}-S_{1}\right) / K_{1}$ is called the stabilized fundamental group. Note that the stabilization procedure does not affect $\pi_{1}\left(\mathbb{C}^{2}-S_{2}\right)=G_{2}$, since $G_{2} \simeq \widetilde{B}_{16} / \operatorname{ker} \tilde{\alpha}$, and $\widetilde{B}_{16}$ is already stabilized.

\subsection{Comparing the BMTs}

In this subsection we prove that the BMF of the branch curves $X_{1}$ and $X_{2}$ are not equivalent. We will do this by looking at the stabilized fundamental groups, related to $X_{1}$ and $X_{2}$. We denote by $K_{2}$ the normal subgroup of $\pi_{1}\left(\mathbb{C}^{2}-S_{2}\right)$ generated 
by all commutators $\left[\gamma_{1}, \gamma_{2}\right], \gamma_{1}, \gamma_{2}$ - geometric generators of $\pi_{1}\left(\mathbb{C}^{2}-S_{2}\right)$, such that $\Theta_{2}\left(\gamma_{1}\right)$ and $\Theta_{2}\left(\gamma_{2}\right)$ are disjoint transpositions (here $\Theta_{2}: \pi_{1}\left(\mathbb{C}^{2}-S_{2}\right) \rightarrow S_{16}$ is the geometric monodromy morphism). As was noted, $\pi_{1}\left(\mathbb{C}^{2}-S_{2}\right) / K_{2}=\pi_{1}\left(\mathbb{C}^{2}-S_{2}\right)$. We also note that $K_{1}=K_{2}$; since it is enough to pick one pair of geometric generators (eg, $x_{2}$ and $\left(x_{2}\right)_{x_{3} x_{1}}$, when the $x_{i}$ are geometric generators), and define $K_{1}=K_{2}=$ $\left\langle\left[x_{2},\left(x_{2}\right)_{x_{3} x_{1}}\right]\right\rangle$.

Theorem 4.15 $G_{1} / K_{1} \not G_{2} / K_{2}$.

Proof Note that $\widetilde{B}_{16} / \operatorname{ker} \tilde{\alpha} \simeq G_{2} \simeq G_{2} / K_{2}$. Denote

$$
G_{2}^{0} \doteq\left(\operatorname{ker}\left(\Theta_{2}: \pi_{1}\left(\mathbb{C}^{2}-S_{2}\right) \rightarrow S_{n}\right) \bigcap \operatorname{ker}\left(\operatorname{deg}: \pi_{1}\left(\mathbb{C}^{2}-S_{2}\right) \rightarrow \mathbb{Z}\right)\right) / K_{2} .
$$

It is known from [18] what is the commutant subgroup of

$$
\widetilde{P}_{n, 0}=\operatorname{ker}\left(\widetilde{B}_{n} \rightarrow S_{n}\right) \bigcap \operatorname{ker}(\operatorname{deg}: \widetilde{B} \rightarrow \mathbb{Z}) .
$$

Explicitly, $\left[\widetilde{P}_{n, 0}, \widetilde{P}_{n, 0}\right]$ is isomorphic to $\mathbb{Z}_{2}$. Therefore, $\left[G_{2}^{0}, G_{2}^{0}\right]$ is a subgroup of $\mathbb{Z}_{2}$, whereas $\left[G_{1}^{0}, G_{1}^{0}\right] \simeq \mathbb{Z}_{2} \oplus \mathbb{Z}_{2}$. But if $G_{1} / K_{1} \simeq G_{2} / K_{2}$ were isomorphic, then these two commutant subgroups would be equal.

Remark 4.16 We believe that an explicit computation of $\pi_{1}\left(\mathbb{C}^{2}-S_{1}\right)$ (as in $[11$; 17]) would have shown that $K_{1}=\{e\}$.

\subsection{Computation for the Galois covers}

Let $\widetilde{\pi}_{i}: \widetilde{X}_{i} \rightarrow \mathbb{C}^{2}$ be the Galois covering corresponding to $\pi_{1}$ (see [11] for definitions). Recall that $\pi_{1}\left(\widetilde{X}_{i}\right)=\operatorname{ker} \Theta_{i} /\left\langle\Gamma_{i, j}^{2}\right\rangle$ where $\Theta_{i}: \pi_{1}\left(\mathbb{C}^{2}-S_{i}\right) \rightarrow S_{n}, \quad n=\operatorname{deg} \pi_{i}(i=$ 1 or 2 ; the degree is the same) and $\left\{\Gamma_{i, j}\right\}$ are the generators of $\pi_{1}\left(\mathbb{C}^{2}-S_{i}\right)$, for $i=1,2$.

In [1] it was proved that $\pi_{1}\left(\widetilde{X}_{1}^{A f f}\right)=\{e\}$. This is also the case for $X_{2}$. We know that the divisibility index of (the embedding of) $X_{2}$ is 1 . Since $G_{2}$ is a quotient of $\widetilde{B}_{16}$, we can now use Liedtke [10, Theorem 4.1] to prove that $\pi_{1}\left(\widetilde{X}_{2}^{A f f}\right)=\{e\}$.

The Main Result Since the stabilized fundamental groups induced from them are not isomorphic, $\varphi_{1}$ is not Hurwitz-equivalent to $\varphi_{2}$. Therefore, $X_{1}$ and $X_{2}$ are not BMT-equivalent. Note that this inequivalence cannot be deduced from the computation of the fundamental groups of the Galois covers, as these groups are isomorphic. 


\section{Appendix: Invariance rules for the BMF of a 5-point}

This appendix shows that the BMF of a 5-point is invariant under certain braids. We focus on the $\operatorname{BMF} \varphi_{2,3}$, where the invariance rules for the other $\varphi_{i, j}(i=1,2,1 \leq$ $j \leq 10)$ are calculated in the same way.

Recall that two factorizations are Hurwitz equivalent if they are obtained from each other by a finite sequence of Hurwitz moves.

Definition (A factorized expression invariant under $h$ ) Let $t=t_{1} \cdots t_{m}$ be a factorized expression in a group $G$. We say that $t$ is invariant under $h \in G$ if $\left(t_{1}\right)_{h}$. $\cdots\left(t_{m}\right)_{h}$ is Hurwitz equivalent to $t_{1} \cdots \cdot t_{m}$.

We recall now a few invariance rules (see [16, section 3]):

Invariance rule II: $Z_{i, j j^{\prime}}^{2}\left(Z_{i i^{\prime}, j j^{\prime}}^{2}\right)$ is invariant under $Z_{j j^{\prime}}^{q}\left(\right.$ resp. $\left.Z_{j j^{\prime}}^{q} Z_{i i^{\prime}}^{p}\right)$.

Invariance rule III: $\quad Z_{i, j j^{\prime}}^{(3)}$ is invariant under $Z_{j j^{\prime}}^{q}$.

For our purposes (see the last paragraph in the proof of Lemma 3.10), it is enough to prove the following proposition.

Proposition $5.1 \varphi_{2,3}$ is invariant under $\left(Z_{11^{\prime}} Z_{33^{\prime}}\right)^{p}\left(Z_{2121^{\prime}} Z_{1616^{\prime}}\right)^{q} \quad \forall p, q \in \mathbb{Z}$.

Proof We first look at the factors outside $\left(F_{3} \cdot\left(F_{3}\right)_{\vartheta}\right)_{\alpha_{3}}$. By the Invariance rule II, the factors $Z_{33^{\prime}, 9}^{2}, Z_{9^{\prime}, 1616^{\prime}}^{2}, \widetilde{Z}_{9^{\prime}, 1616^{\prime}}^{2}, \widetilde{Z}_{33^{\prime}, 9}^{2}$ are invariant under $Z_{33^{\prime}}$ and $Z_{1616^{\prime}}$; by ([16, invariance remark (iv)]), these factors are also invariant under $Z_{11^{\prime}}$ and $Z_{2121^{\prime}}$ (since the paths are disjoint). Again, by the same invariance remark, $\widetilde{Z}_{9,9^{\prime}}$ is invariant under $Z_{i i^{\prime}} i=1,3,16,21$. By the Invariance rule III, the factors $Z_{11^{\prime}, 9}^{(3)}$ and $\bar{Z}_{9,2121^{\prime}}^{(3)}$ are invariant under $Z_{11^{\prime}}$ and $Z_{2121^{\prime}}$ (and also under $Z_{33^{\prime}}$ and $Z_{1616^{\prime}}$ by the invariance remark (iv)).

We note that the conjugation by the braid $Z_{\alpha_{3}}$ is actually conjugation by $Z_{33^{\prime}, 9}^{2} Z_{11^{\prime}, 9}^{2}$, so it is also invariant under $Z_{i i^{\prime}} i=1,3,16,21$ (by invariance rule II and remark (iv)). When looking at the expression $F_{3} \cdot\left(F_{3}\right)_{\vartheta}$, we see that this case was already done in [3, invariance property 8.7]; it was proved there that $F_{3} \cdot\left(F_{3}\right)_{\vartheta}$ is invariant under $\left(Z_{11^{\prime}} Z_{33^{\prime}}\right)^{p}\left(Z_{2121^{\prime}} Z_{1616^{\prime}}\right)^{q}$.

\section{References}

[1] M Amram, C Ciliberto, R Miranda, M Teicher, Braid monodromy factorization for a non-prime K3 surface branch curve, to appear in Israel Journal of Mathematics 
[2] M Amram, M Friedman, M Teicher, The fundamental group of complement of a branch curve of a Hirzebruch surface $F_{2,(2,2)}$, submitted to Topology

[3] M Amram, M Teicher, The fundamental group of the complement of the branch curve of the double torus, Journal of Mathematics 40 (2003) 587-893

[4] D Auroux, S K Donaldson, L Katzarkov, M Yotov, Fundamental groups of complements of plane curves and symplectic invariants, Topology 43 (2004) 1285-1318 MR2081427

[5] C Ciliberto, R Miranda, On the Gaussian map for canonical curves of low genus, Duke Math. J. 61 (1990) 417-443 MR1074304

[6] C Ciliberto, R Miranda, M Teicher, Pillow degenerations of $K 3$ surfaces, from: "Applications of algebraic geometry to coding theory, physics and computation (Eilat, 2001)", NATO Sci. Ser. II Math. Phys. Chem. 36, Kluwer Acad. Publ., Dordrecht (2001) 53-63 MR1866895

[7] M Friedman, M Teicher, On the fundamental group related to the Hirzebruch surface $F_{1}$, to appear in Sci. China ser. A.

[8] V S Kulikov, M Teicher, Braid monodromy factorizations and diffeomorphism types, Izv. Ross. Akad. Nauk Ser. Mat. 64 (2000) 89-120 MR1770673

[9] E Liberman, M Teicher, The Hurwitz equivalence problem is undecidable arXiv: math.LO/0511153

[10] C Liedtke, On fundamental groups of Galois closures of generic projections, Bonner Mathematische Schriften [Bonn Mathematical Publications], 367, Universität Bonn Mathematisches Institut, Bonn (2004) MR2206239 Dissertation, Rheinische FriedrichWilhelms-Universität Bonn, Bonn, 2004

[11] B Moishezon, On cuspidal branch curves, J. Algebraic Geom. 2 (1993) 309-384 MR1203688

[12] B Moishezon, A Robb, M Teicher, On Galois covers of Hirzebruch surfaces, Math. Ann. 305 (1996) 493-539 MR1397434

[13] B Moishezon, M Teicher, Simply-connected algebraic surfaces of positive index, Invent. Math. 89 (1987) 601-643 MR903386

[14] B Moishezon, M Teicher, Braid group technique in complex geometry. I. Line arrangements in $\mathbf{C P}^{2}$, from: "Braids (Santa Cruz, CA, 1986)", Contemp. Math. 78, Amer. Math. Soc., Providence, RI (1988) 425-555 MR975093

[15] B Moishezon, M Teicher, Braid group technique in complex geometry. II. From arrangements of lines and conics to cuspidal curves, from: "Algebraic geometry (Chicago, IL, 1989)", Lecture Notes in Math. 1479, Springer, Berlin (1991) 131-180 MR1181212 
[16] B Moishezon, M Teicher, Braid group techniques in complex geometry. IV. Braid monodromy of the branch curve $S_{3}$ of $V_{3} \rightarrow \mathbf{C P}^{2}$ and application to $\pi:\left(\mathbf{C P}^{2}-S_{3}, *\right)$, from: "Classification of algebraic varieties (L'Aquila, 1992)", Contemp. Math. 162, Amer. Math. Soc., Providence, RI (1994) 333-358 MR1272707

[17] B Moishezon, M Teicher, Braid group technique in complex geometry. V. The fundamental group of a complement of a branch curve of a Veronese generic projection, Comm. Anal. Geom. 4 (1996) 1-120 MR1393558

[18] A Robb, The topology of branch curves of complete intersections, $\mathrm{PhD}$ thesis, Columbia university (1994)

[19] A Robb, On branch curves of algebraic surfaces, from: "Singularities and complex geometry (Beijing, 1994)”, AMS/IP Stud. Adv. Math. 5, Amer. Math. Soc., Providence, RI (1997) 193-221 MR1468277

[20] E R Van Kampen, On the Fundamental Group of an Algebraic Curve, Amer. J. Math. 55 (1933) 255-267 MR1506962

Department of Mathematics, Bar-Ilan University, 52900 Ramat Gan, Israel.

teicher@macs.biu.ac.il, fridmam@macs.biu.ac.il

http://www.cs.biu.ac.il/ teicher/

Received: 1 August 2007 Revised: 27 December 2007

Algebraic $\& \mathcal{G}$ Geometric Topology, Volume 8 (2008) 